\title{
An integral formulation for steady-state elastoplastic contact over a coated half-plane ${ }^{\star}$
}

\author{
Chunying Dong ${ }^{\star \star}$, Marc Bonnet \\ Laboratoire de Mécanique des Solides, Ecole Polytechnique, Palaiseau, France. \\ e-mail: bonnet@lms.polytechnique.fr
}

The date of receipt and acceptance will be inserted by the editor

\begin{abstract}
A boundary-domain integral equation for a coated half-space (elastically isotropic homogeneous substratum, possibly anisotropic coating layer) is developed. The half-space fundamental solution is used, so that the discretization is limited to the potential contact zone (boundary elements), the potentially plastic part of the substratum and the coating layer (domain integration cells). Steady-state elastoplastic analysis is implemented within this framework, for plane-strain conditions, for solving rolling and/or sliding contact problems, where at the moment the contact load comes from either a purely elastic contact analysis or is of Hertz type. The constitutive integration is of implicit type. In order to improve accuracy and computational efficiency, infinite elements are used. Comparison of numerical results with other sources, when available, is satisfactory. The present formulation is also used to compute the contact pressure for an isotropic (or anisotropic) coating on an isotropic homogeneous half-space indented by an elastic punch.
\end{abstract}

Key words boundary integral equation - coated half-space - steady-state elastoplastic analysis - implicit constitutive integration.

\section{Introduction}

The study of rolling and/or sliding of hard cylinders on semi-infinite elastoplastic regions having either elastic- perfect plastic or kinematically hardening constitutive properties goes back to Merwin and Johnson (1963) and Johnson and Jefferis (1963) who used simplifying assumptions such as a Hertzian contact

* Computational Mechanics, 28:105-121 (2002)

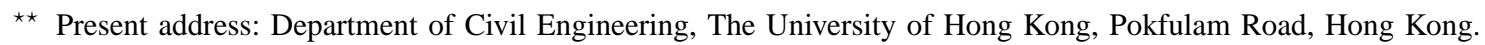
e-mail: cdong@hkucc.hku.hk

Correspondence to: Marc Bonnet, Laboratoire de Mécanique des Solides, Ecole Polytechnique, F-91128 Palaiseau cedex, France 
pressure distribution and equivalence between the total strain cycle and the elastic strain cycle. In order to avoid those assumptions, Bhargava, Hahn, and Rubin (1985a,b) adopted a finite element formulation in a fixed frame and a traditional time-stepping scheme. This approach is time-consuming because of the need to deal with incrementally moving loads. Yu, Moran, and Keer (1993) extended the direct approach proposed by Zarka and Casier (1979) to investigate the steady-state problem under repeated rolling loading. This method is very effective in solving cyclic rolling contact problem with linear-hardening materials and was also used to study ratchetting behavior (Sakae and Keer, 1997) by adopting a nonlinear kinematic hardening rule proposed by Armstrong and Frederick (1966). Dang Van and Maitournam (1993) proposed an efficient and reliable steady-state algorithm for the calculation of stresses and strains in the half-space with perfect plastic or linear kinematic hardening materials. However, although the underlying steady-state assumptions imply that the computational domain is in principle unbounded in the direction of the moving load, finite element-based approches require bounded meshes in practice. This is a significant shortcoming, especially in view of the fact that plastic strains are expected to develop up to infinity. The characteristic length of the computational region must thus be much larger than that of the contact area, and the boundary conditions to apply at infinity are not clear.

On the other hand, boundary element method (BEM) is a very good method for solving elastic problems, especially with unbounded domains. Besides, it still has some advantages for problems with small plastic regions. For problems with elastoplastic behavior under repeated rolling loading, the plastic regions develop near the surface. Hence, BEM is expected to provide an efficient tool for the analysis of the stresses and strains in half space under cyclic loading.

The application of BEM to steady-state elastoplastic rolling contact problems was first proposed in Lederer, Bonnet, and Maitournam (1998), where a regularized integral equation formulation for contact problems on homogeneous elastoplastic bodies was used, together with an implicit elastoplastic constitutive integration algorithm. The presence and effect of a coating, often used to extend the fatigue life of various components, was not considered.

This paper extends the approach of Lederer, Bonnet, and Maitournam (1998) to rolling/sliding contact on a coated half-space. The boundary-domain traction and strain integral equations are still based on the half-space fundamental solutions, but here a new singular domain integral (over the layer) arises due to the contrast of elastic constants. These highly singular integral equations require a specific regularization treatment, presented in section 3. The present formulation is tailored for half-space geometries: the discretization is limited to the potential contact zone (boundary elements), the potentially plastic part of the substratum and the coating layer (domain integration cells). Steady-state elastoplastic analysis is implemented within this framework, for linear-kinematic-hardening constitutive plastic behavior. The integration with respect to load step is of implicit type. For simplicity and following a practice commonly used for this 
type of analysis, the elastic-plastic analysis is carried out for a given contact load, either of Hertz type or coming from a purely elastic contact calculation (in other words, elastoplasticity and contact are treated in an uncoupled fashion). The present formulation can be also used to compute the (a priori unknown) contact pressure for an isotropic (or anisotropic) coating on an isotropic homogeneous half-space indented by an elastic roller. These various implementation issues are discussed in section 4 . In order to test numerically the proposed boundary-domain integral equation for a coated half-space in 2-D plane strain, numerical examples are presented in section 5 for stress analysis in elastic contact, elastoplastic analysis under a statical Hertz load and steady-state elastoplastic rolling under a moving Hertz load. The influence of the coating and the friction coefficient on contact pressure is studied. Comparisons with other published results are made when possible.

\section{Geometry and basic governing equations}

The generic configuration considered in this paper (figure 1) is a coated half-space $\Omega=\left\{x_{1} \geq 0\right\}$ : a coating $\Omega^{c}$ of constant thickness $h$ and made of possibly anisotropic material lies on top of a substratum $\Omega^{s}=\Omega-\Omega^{c}$ made of isotropic material. A given loading is applied on a bounded subset $\Gamma_{a}$ of the boundary $\Gamma=\left\{x_{1}=0\right\}$ while the complementary boundary $\Gamma-\Gamma_{a}$ is traction-free. Perfect bonding is assumed along the interface $\Gamma_{i}$ between the coating and the substratum. For the numerical results presented in section 5, an elastic-plastic constitutive behavior is considered for the substratum, whereas the coating is assumed to remain purely elastic. However, for the sake of completeness, the integral equations are presented in section 3 assuming that plastic strains are present in both the coating and the substratum.

The stresses $\sigma^{c}$ in $\Omega^{c}$ and $\sigma$ in $\Omega^{s}$ solve the equilibrium field equation without body forces, i.e.:

$$
\operatorname{div} \boldsymbol{\sigma}=\mathbf{0} \quad \text { in } \Omega^{c}, \Omega^{s}
$$

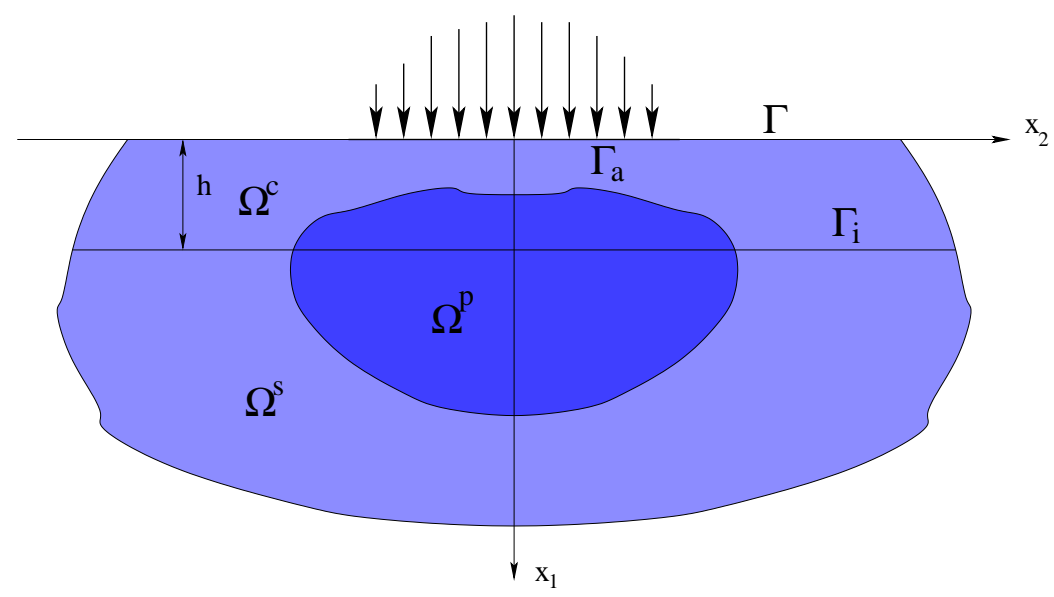

Fig. 1 A coated half-space 
together with the boundary conditions ( $\boldsymbol{n}$ : outer unit normal to $\Gamma$ )

$$
\begin{array}{ll}
\boldsymbol{\sigma}^{c} \cdot \boldsymbol{n}=\boldsymbol{t} & \text { on } \Gamma_{a} \\
\boldsymbol{\sigma}^{c} \cdot \boldsymbol{n}=\mathbf{0} & \text { on } \Gamma-\Gamma_{a}
\end{array}
$$

and perfect bonding conditions along the interface $\Gamma_{i}$ between the coating and the substratum $\left(\boldsymbol{u}^{c}\right.$ and $\boldsymbol{u}$ : displacement in $\Omega^{c}$ and $\Omega^{s}$ ):

$$
\left\{\begin{aligned}
\left(\boldsymbol{\sigma}^{c}-\boldsymbol{\sigma}\right) \cdot \boldsymbol{n} & =\mathbf{0} \\
\boldsymbol{u}^{c}-\boldsymbol{u} & =\mathbf{0}
\end{aligned} \quad \text { on } \Gamma_{i}\right.
$$

The strains $\varepsilon$, stresses $\sigma$ and plastic strains $\hat{\varepsilon}$ are related through the constitutive equations

$$
\boldsymbol{\sigma}=\boldsymbol{L}:(\varepsilon-\hat{\varepsilon})
$$

in the substratum, and

$$
\boldsymbol{\sigma}^{c}=\boldsymbol{L}^{c}:\left(\varepsilon^{c}-\hat{\boldsymbol{\varepsilon}}^{c}\right)
$$

in the coating. Isotropic elasticity is assumed in the substratum, so that the corresponding elasticity tensor $\boldsymbol{L}$ has the form:

$$
L_{i j k \ell}=G\left[\frac{2 \nu}{1-2 \nu} \delta_{i j} \delta_{k \ell}+\left(\delta_{i k} \delta_{j \ell}+\delta_{i \ell} \delta_{j k}\right)\right]
$$

( $G$ : shear modulus, $\nu$ : Poisson ratio), where $\delta_{i j}$ is the Kronecher symbol. On the other hand, the elastic properties of the coating are possibly anisotropic, and the corresponding elasticity tensor is expressed as $\boldsymbol{L}^{c}=\boldsymbol{L}-\Delta \boldsymbol{L}$, where $\Delta \boldsymbol{L}$ denotes the (possibly anisotropic) contrast of elastic properties between the coating and the substratum. In addition, rate-independent plasticity with the Von Mises yield criterion and an associated flow rule is assumed (see section 4.2 for details about the corresponding constitutive equations).

In this paper, two kinds of situations are considered: fixed loads (relevant for modelling e.g. indentation experiments) and loads moving at a constant velocity $V$ along the horizontal $x_{2}$-direction (for modelling repeated rolling / sliding contact). In the latter case (referred to as steady-state), following the approach of Dang Van and Maitournam (1993), a frame moving along with the load is introduced, i.e. a new coordinate $\hat{x}_{2}=x_{2}-V t$ is defined so that all physical quantities are time-independent in the $\left(x_{1}, \hat{x}_{2}\right)$ coordinates. Letting $X$ denote one such quantity, its particle time derivative is thus given by:

$$
\frac{d X}{d t}=-V \frac{\partial X}{\partial \hat{x}_{2}}
$$

This approach is significantly more efficient than a more traditional incremental solution strategy in a fixed frame, used in e.g. Bhargava, Hahn, and Rubin (1985a,b).

Here, and in Lederer, Bonnet, and Maitournam (1998) for homogeneous half-planes, the novel feature consists in using an integral equation approach for dealing with steady-state problems, whereas Dang Van and Maitournam (1993) used the finite element method (FEM). Steady-state problems entail considering 
domains that are infinite in the $x_{2}$-direction, due to the underlying requirement of translational invariance. Infinite media are well handled by integral equation formulations: decaying conditions at infinity are built in these formulations, and other conditions at infinity can be considered as well without giving rise to divergent integrals. On the other hand, FEM for steady-state calculations requires a bounded computational domain, because nonzero (but asymptotically constant) plastic strains are expected at infinity, which prevents one to use infinite elements (divergent integrals at infinity do arise in that case).

\section{Integral representation of displacement and strain in a coated half-space}

The equilibrium of the coated half-space is formulated in terms of boundary-domain integral equations. The usual basis for such formulation is a reciprocity identity between the unknown state and a known fundamental solution, here chosen to be an elastic field generated by an unit point force applied at a fixed source point $P$ and along a fixed direction $k$ in a fictitious body endowed with the isotropic elastic moduli of the substratum elastic constants. The components $u_{i}$ of the unknown displacement field are governed by the equilibrium equation

$$
L_{i j a b}^{c}\left(u_{a, b j}-\hat{\varepsilon}_{a b, j}\right)=0
$$

(commas denoting partial differentiations w.r.t. coordinates of the field point $q$ ) while the components $U_{i k}(P, q)$ of the fundamental displacement are governed by the equation:

$$
L_{i j a b} U_{a k, b j}+\delta(q-P) \delta_{i k}=0
$$

where $\delta(q-P)$ is the Dirac distribution at point $P$. Multiplying Eq. (8) by $U_{i k}(P, q)$ and Eq. (9) by $u_{i}$, integrating both equations over a domain $\Omega$, invoking the defining property of $\delta(q-P)$, integrating by parts the remaining integrals and subtracting the resulting identities, one obtains the reciprocity identity:

$$
\begin{aligned}
\kappa(P) u_{k}(P)= & \int_{\partial \Omega}\left\{U_{i k}(P ; q) t_{i}(q)-T_{i k}(P ; q) u_{i}(q)\right\} \mathrm{d} \Gamma(q) \\
& +\int_{\Omega}\left(\Sigma_{k a b}(P, Q)-\Delta L_{i j a b} U_{i k, j}(P, Q)\right) \hat{\varepsilon}_{a b}(Q) \mathrm{d} \Omega(Q) \\
& +\int_{\Omega} \Delta L_{i j a b} U_{i k, j}(P, Q) u_{i, j}(Q) \mathrm{d} \Omega(Q)
\end{aligned}
$$

where $\kappa(P)=1$ or 0 according to whether the source point $P$ is interior or exterior to the domain $\Omega$; besides, $t_{i}, T_{i k}$ and $\Sigma_{i j k}$ denote the components of unknown tractions, fundamental tractions and fundamental stresses, respectively.

The formulation presented in this section relies upon the use of the fundamental solution which is traction-free on $\Gamma$. It has been implemented for two-dimensional (plane-strain) calculations, using the fundamental solution for a traction-free half-plane (see Telles et al., 1981), but essentially holds for three- 
dimensional situations as well, using the half-space Mindlin fundamental solution. From Eq. (10), the equilibrium of the coating $\Omega^{c}$ considered in isolation is thus governed by:

$$
\begin{aligned}
\kappa_{c}(P) u_{k}(P)= & \int_{\Gamma_{a}} U_{i k}(P ; q) t_{i}(q) \mathrm{d} \Gamma(q)+\int_{\Gamma_{i}}\left\{U_{i k}(P ; q) t_{i}(q)-T_{i k}(P ; q) u_{i}(q)\right\} \mathrm{d} \Gamma(q) \\
& +\int_{\Omega^{c}}\left(\Sigma_{k a b}(P, Q)-\Delta L_{i j a b} U_{i k, j}(P, Q)\right) \hat{\varepsilon}_{a b}(Q) \mathrm{d} \Omega(Q) \\
& +\int_{\Omega^{c}} \Delta L_{i j a b} U_{i k, j}(P, Q) u_{i, j}(Q) \mathrm{d} \Omega(Q)
\end{aligned}
$$

The equilibrium of the substratum $\Omega^{s}$ considered in isolation is governed by:

$$
\kappa_{s}(P) u_{k}(P)=\int_{\Gamma_{i}}\left\{U_{i k}(P ; q) t_{i}(q)-T_{i k}(P ; q) u_{i}(q)\right\} \mathrm{d} \Gamma(q)+\int_{\Omega^{s}} \Sigma_{i j k}(P, Q) \hat{\varepsilon}_{i j}(Q) \mathrm{d} \Omega(Q)
$$

since in that case $\Delta \boldsymbol{L}=\mathbf{0}$. Note that in the last two equations the tractions refer to the unit normal vector which is exterior to the coating and the substratum, respectively.

Next, Eqs. (11) and (12) are added. The integrals over the interface $\Gamma_{i}$ cancel out in the process by virtue of the bonding conditions (4); besides, one has $\kappa_{c}(P)+\kappa_{s}(P)=1$ for any source point in the half-space. The displacement at any point $P$ in the coated half-space is thus given by:

$$
\begin{aligned}
& u_{k}(P)=\int_{\Gamma_{a}} U_{i k}(P ; q) t_{i}(q) \mathrm{d} \Gamma(q) \\
& \quad+\int_{\Omega^{c}}\left(L_{i j m \ell}^{c} \hat{\varepsilon}_{m \ell}(Q)+\Delta L_{i j m \ell} \varepsilon_{m \ell}(Q)\right) E_{i j k}(P, Q) \mathrm{d} \Omega(Q)+\int_{\Omega^{s}} \Sigma_{i j k}(P, Q) \hat{\varepsilon}_{i j}(Q) \mathrm{d} \Omega(Q)
\end{aligned}
$$

where

$$
E_{i j k}=\frac{1}{2}\left(U_{i k, j}+U_{k j, i}\right)=\frac{1}{2 G}\left(\Sigma_{i j k}-\chi \Sigma_{k a a} \delta_{i j}\right)
$$

and $\chi=\nu /(1+\nu)$ (in three dimensions) or $\chi=\nu$ (in plane strain).

Note that (13) is written in terms of the fundamental solutions for a half-plane with homogeneous and isotropic elastic moduli. It involves domain integrals over the whole coating (in practice very thin) and over the potentially plastic part of the substratum. A possible alternative would be to use the fundamental solutions for coated half-spaces (Pan, Chen, and Amadei, 1997). However, the latter are much more complicated (and computationally intensive); besides, the discretization of the coating is necessary anyway if plastic strains are expected to develop in it.

Equation (13) is valid for any source point $P$ in $\Omega$ or on $\Gamma$; in particular the well-known properties of the fundamental solution (namely that $U_{i k}=O(\ln r)$ and $U_{i k, j}, \Sigma_{i j k}=O(1 / r)$ ) ensure that all integrals are convergent in the ordinary sense. Assuming that the loading on $\Gamma_{a}$ is prescribed, the only unknowns in Eq. (13) are the plastic strains in $\hat{\Omega}$ and the total strains in $\Omega^{c}$.

To find these quantities, as usual for elastic-plastic calculations based on an integral equation approach (see Mukherjee and Chandra, 1987), the integral representation for the strain tensor is also needed. Potentially non-integrable singularities arise in the differentiation of Eq. (13) w.r.t the coordinates of $P$, so that 
this operation cannot be carried out straightforwardly. Here the indirect regularization approach (see e.g. (Tanaka, Sladek, and Sladek, 1994; Bonnet, 1999)) is followed. To this end, assume that $P$ lies inside either the substratum or the coating (i.e. not on the interface) and let $\Omega_{e}$ denote a portion of $\Omega^{s}$ or $\Omega^{c}$ containing $P$ as an interior point (in practice, $\Omega_{e}$ would typically be the integration cell containing $P$ ). Since:

$$
\int_{\Omega^{s}} U_{i k, j}(P, Q) \mathrm{d} \Omega(Q)=\int_{\Gamma_{e}} U_{i k}(P, q) n_{j}(q) \mathrm{d} \Gamma(q)
$$

one has for any constant symmetric tensor $s$ :

$$
0=s_{i j}\left\{\int_{\Omega_{e}} E_{i j k}(Q) \mathrm{d} \Omega(Q)-\int_{\Gamma_{e}} U_{i k}(Q) n_{j}(q) \mathrm{d} \Gamma(q)\right\}
$$

First, let $P$ lie in $\Omega^{s}$. Putting $s=\boldsymbol{L}: \hat{\varepsilon}(P)$ in Eq. (15) and subtracting the resulting identity from Eq. (13), one obtains:

$$
\begin{aligned}
u_{k}(P)= & \int_{\Gamma_{a}} U_{i k}(P ; q) t_{i}(q) \mathrm{d} \Gamma(q)+\left(L_{i j a b} \hat{\varepsilon}_{a b}(P)\right) \int_{\Gamma_{e}} U_{i k}(P ; q) n_{j}(q) \mathrm{d} \Gamma(q) \\
& +\int_{\Omega^{c}}\left(L_{i j a b}^{c} \hat{\varepsilon}_{a b}(Q)+\Delta L_{i j a b} \varepsilon_{a b}(Q)\right) E_{i j k}(P, Q) \mathrm{d} \Omega(Q) \\
& +\int_{\Omega^{s}-\Omega_{e}} \Sigma_{i j k}(P, Q) \hat{\varepsilon}_{i j}(Q) \mathrm{d} \Omega(Q)+\int_{\Omega_{e}} \Sigma_{i j k}(P, Q)\left(\hat{\varepsilon}_{i j}(Q)-\hat{\varepsilon}_{i j}(P)\right) \mathrm{d} \Omega(Q)
\end{aligned}
$$

The integral representation formula in the above form may be safely differentiated w.r.t. the generic $\ell$ coordinate of $P$ since this operation gives rise to convergent integrals only. One obtains:

$$
\begin{aligned}
u_{k, \ell}(P)= & \int_{\Gamma_{a}} t_{i}(q) U_{i k, \bar{\ell}}(P, q) \mathrm{d} \Gamma(q)+\left(L_{i j a b} \hat{\varepsilon}_{a b}(P)\right) \int_{\Gamma_{e}} n_{j}(q) U_{i k, \bar{\ell}}(P, q) \mathrm{d} \Gamma(q) \\
& +\int_{\Omega^{c}}\left(L_{i j a b}^{c} \hat{\varepsilon}_{a b}(Q)+\Delta L_{i j a b} \varepsilon_{a b}(Q)\right) E_{i j k, \bar{\ell}}(P, Q) \mathrm{d} \Omega(Q) \\
& +\int_{\Omega^{s}-\Omega_{e}} \Sigma_{i j k, \bar{\ell}}(P, Q) \hat{\varepsilon}_{i j}(Q) \mathrm{d} \Omega(Q)+\int_{\Omega_{e}} \Sigma_{i j k, \bar{\ell}}(P, Q)\left(\hat{\varepsilon}_{i j}(Q)-\hat{\varepsilon}_{i j}(P)\right) \mathrm{d} \Omega(Q) \\
& +L_{i j a b} \hat{\varepsilon}_{a b, \bar{\ell}}(P)\left\{\int_{\Gamma_{e}} n_{j} U_{i k}(P, q) \mathrm{d} \Gamma(q)-\int_{\Omega_{e}} E_{i j k}(P, Q) \mathrm{d} \Omega(Q)\right\}
\end{aligned}
$$

(the overbar in ()$_{, \bar{\ell}}$ denoting a partial derivative w.r.t. the $\ell$-coordinate of $P$ ). Upon noting that the last line vanishes due to equation (15), the strains at the source point $P$ can be expressed as

$$
\begin{aligned}
\varepsilon_{k \ell}(P)= & \int_{\Gamma_{a}} t_{i}(q) U_{k \ell i}^{\star}(P, q) \mathrm{d} \Gamma(q)+\left(L_{i j a b} \hat{\varepsilon}_{a b}(P)\right) \int_{\Gamma_{e}} n_{j}(q) U_{k \ell i}^{\star}(P, q) \mathrm{d} \Gamma(q) \\
& +\int_{\Omega^{c}}\left(L_{i j a b}^{c} \hat{\varepsilon}_{a b}(Q)+\Delta L_{i j a b} \varepsilon_{a b}(Q)\right) E_{k \ell i j}^{\star}(P, Q) \mathrm{d} \Omega(Q) \\
& +\int_{\Omega^{s}-\Omega_{e}} \sum_{k \ell i j}^{\star}(P, Q) \hat{\varepsilon}_{i j}(Q) \mathrm{d} \Omega(Q)+\int_{\Omega_{e}} \Sigma_{k \ell i j}^{\star}(P, Q)\left(\hat{\varepsilon}_{i j}(Q)-\hat{\varepsilon}_{i j}(P)\right) \mathrm{d} \Omega(Q)
\end{aligned}
$$

having put:

$$
\begin{aligned}
U_{k \ell i}^{\star}(P, Q) & =\frac{1}{2}\left(U_{i k, \bar{\ell}}(P, Q)+U_{i \ell, \bar{k}}(P, Q)\right) \\
\Sigma_{k \ell i j}^{\star}(P, Q) & =\frac{1}{2}\left(\Sigma_{i j k, \bar{\ell}}(P, Q)+\Sigma_{i j \ell, \bar{k}}(P, Q)\right) \\
E_{k \ell i j}^{\star}(P, Q) & =\frac{1}{2}\left(E_{i j k, \bar{\ell}}(P, Q)+E_{i j \ell, \bar{k}}(P, Q)\right)
\end{aligned}
$$


The expressions for $U_{i k}, \Sigma_{i j k}, U_{k \ell i}^{\star}$ and $\Sigma_{k \ell i j}^{\star}$ are available Telles and Brebbia (1981) (for plane-strain problems) or in Balas, Sladek, and Sladek (1989) (for three-dimensional problems). Those for $E_{k \ell i j}^{\star}$ follow by virtue of (14).

Now let $P$ lie in $\Omega^{c}$. Putting $s=\boldsymbol{L}^{c}: \hat{\varepsilon}(P)+\Delta \boldsymbol{L}: \varepsilon(P)$ in Eq. (15) and subtracting the resulting identity from Eq. (13), the regularized form of Eq. (13) is obtained as:

$$
\begin{aligned}
u_{k}(P)= & \int_{\Gamma_{a}} U_{i k}(P ; q) t_{i}(q) \mathrm{d} \Gamma(q)+\left(\Delta L_{i j a b} \varepsilon_{a b}(P)+L_{i j a b}^{c} \hat{\varepsilon}_{a b}(P)\right) \int_{\Gamma_{e}} n_{j}(q) U_{i k}(P, q) \mathrm{d} \Gamma(q) \\
& +\int_{\Omega^{c}-\Omega_{e}}\left(L_{i j a b}^{c} \hat{\varepsilon}_{a b}(Q)+\Delta L_{i j a b} \varepsilon_{a b}(Q)\right) E_{i j k}(P, Q) \mathrm{d} \Omega(Q) \\
& +\int_{\Omega_{e}}\left\{L_{i j a b}^{c}\left(\hat{\varepsilon}_{a b}(Q)-\hat{\varepsilon}_{a b}(P)\right)+\Delta L_{i j a b}\left(\varepsilon_{a b}(Q)-\varepsilon_{a b}(P)\right)\right\} E_{i j k}(P, Q) \mathrm{d} \Omega(Q) \\
& +\int_{\Omega^{s}} \Sigma_{i j k}(P, Q) \hat{\varepsilon}_{i j}(Q) \mathrm{d} \Omega(Q)
\end{aligned}
$$

Again, it is now safe to differentiate equation (19) with respect to the $\ell$-coordinate of $P$. This results in:

$$
\begin{aligned}
u_{k, \ell}(P)= & \int_{\Gamma_{a}} U_{i k, \bar{\ell}}(P ; q) t_{i}(q) \mathrm{d} \Gamma(q)+\left(\Delta L_{i j a b} \varepsilon_{a b}(P)+L_{i j a b}^{c} \hat{\varepsilon}_{a b}(P)\right) \int_{\Gamma_{e}} n_{j}(q) U_{i k, \bar{\ell}}(P, q) \mathrm{d} \Gamma(q) \\
& +\int_{\Omega^{c}-\Omega_{e}}\left(L_{i j a b}^{c} \hat{\varepsilon}_{a b}(Q)+\Delta L_{i j a b} \varepsilon_{a b}(Q)\right) E_{i j k, \bar{\ell}}(P, Q) \mathrm{d} \Omega(Q) \\
& +\int_{\Omega_{e}}\left\{L_{i j a b}^{c}\left(\hat{\varepsilon}_{a b}(Q)-\hat{\varepsilon}_{a b}(P)\right)+\Delta L_{i j a b}\left(\varepsilon_{a b}(Q)-\varepsilon_{a b}(P)\right)\right\} E_{i j k, \bar{\ell}}(P, Q) \mathrm{d} \Omega(Q) \\
& +\int_{\Omega^{s}} \Sigma_{i j k, \bar{\ell}}(P, Q) \hat{\varepsilon}_{i j}(Q) \mathrm{d} \Omega(Q) \\
& +\left(\Delta L_{i j a b} \varepsilon_{a b, \bar{\ell}}(P)+L_{i j a b}^{c} \hat{\varepsilon}_{a b, \bar{\ell}}(P)\right) \\
& \left\{\int_{\Gamma_{e}} n_{j}(q) U_{i k}(P, q) \mathrm{d} \Gamma(q)-\int_{\Omega_{e}} E_{i j k}(P, Q) \mathrm{d} \Omega(Q)\right\}
\end{aligned}
$$

where again the term in curly brackets in the last line vanishes due to equation (15). Thus the strains at the source point $P$ can be expressed as

$$
\begin{aligned}
\varepsilon_{k \ell}(P)= & \int_{\Gamma_{a}} U_{k \ell i}^{\star}(P, q) t_{i}(q) \mathrm{d} \Gamma(q)+\left(\Delta L_{i j a b} \varepsilon_{a b}(P)+L_{i j a b}^{c} \hat{\varepsilon}_{a b}(P)\right) \int_{\Gamma_{e}} n_{j}(q) U_{k \ell i}^{\star}(P, q) \mathrm{d} \Gamma(q) \\
& +\int_{\Omega^{c}-\Omega_{e}}\left(L_{i j a b}^{c} \hat{\varepsilon}_{a b}(Q)+\Delta L_{i j a b} \varepsilon_{a b}(Q)\right) E_{k \ell i j}^{\star}(P, Q) \mathrm{d} \Omega(Q) \\
& +\int_{\Omega_{e}}\left\{L_{i j a b}^{c}\left(\hat{\varepsilon}_{a b}(Q)-\hat{\varepsilon}_{a b}(P)\right)+\Delta L_{i j a b}\left(\varepsilon_{a b}(Q)-\varepsilon_{a b}(P)\right)\right\} E_{k \ell i j}^{\star}(P, Q) \mathrm{d} \Omega(Q) \\
& +\int_{\Omega^{s}} \Sigma_{k \ell i j}^{\star}(P, Q) \hat{\varepsilon}_{i j}(Q) \mathrm{d} \Omega(Q)
\end{aligned}
$$

In equations (18) and (21), all integrals except those over $\Omega_{e}$ are nonsingular. Assuming that $\hat{\varepsilon}$ and $\varepsilon$ have $C^{0, \alpha}$ smoothness at $q=P$, the integrands in integrals over $\Omega_{e}$ are $O\left(r^{-2+\alpha}\right)$ are weakly singular and can be evaluated by appropriate numerical integration methods (see for example Mustoe, 1984).

Equations (13), (18) and (21) can be applied to either three-dimensional or two-dimensional situations, using the appropriate fundamental solutions and ranges of indices. 


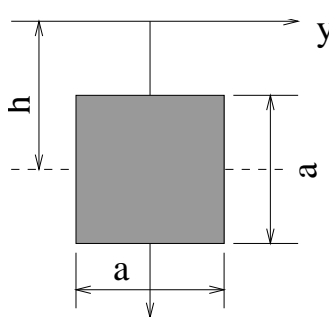

$\mathrm{X}$

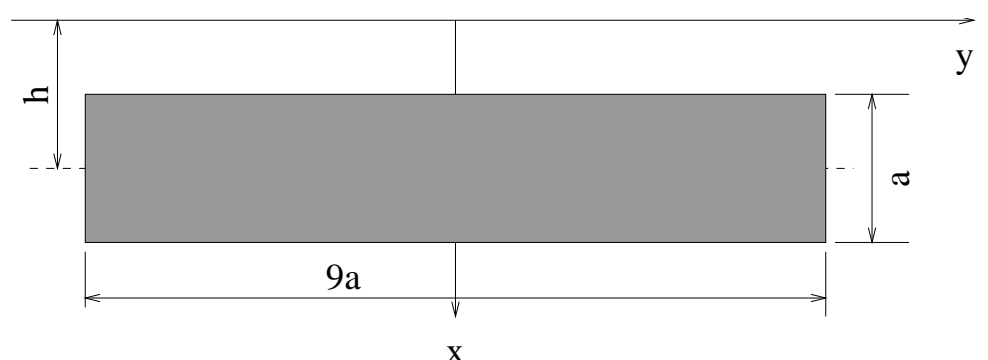

(b)

Fig. 2 Plastic inclusions in the half-space

The correctness of Eqs. (18) and (21) can be checked against closed-form expressions of the stress field produced in a linear isotropic elastic half-space under plane-strain conditions by a rectangular inclusion with constant plastic strain, established by Ballard and Constantinescu (1994). Material constants are $E=$ $210 \mathrm{GPa}$ and $\nu=0.3$. Figures 3 and 4 show that stresses obtained using Eqs. (18) and (21) agree well with the corresponding analytical values, for the inclusions described by Figure 2 (a) and (b), respectively, with $\hat{\varepsilon}_{x x}=1 ., \hat{\varepsilon}_{y y}=-0.5$, and $\hat{\varepsilon}_{x y}=0$. and using $E=210 \mathrm{GPa}$ and $\nu=0.3$.

\section{Numerical implementation}

An implementation of the approach of Secs. 2 and 3 under plane-strain conditions is now presented.

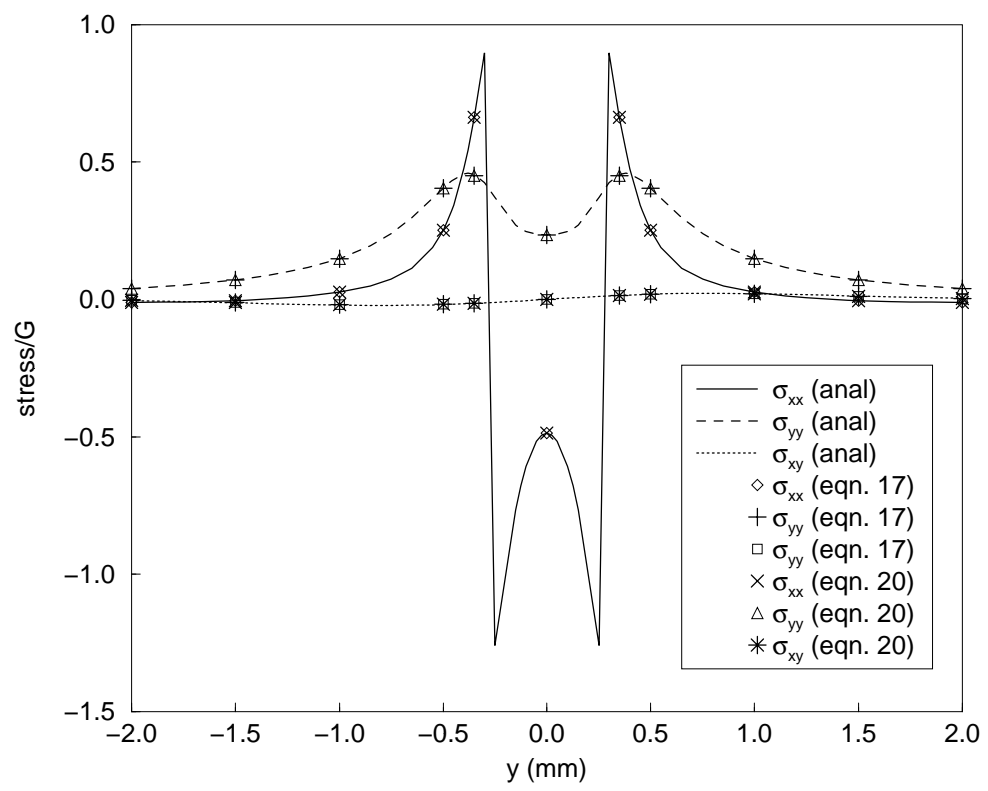

Fig. 3 Stresses produced by the inclusion of figure 2(a): comparison of analytical and numerical values 


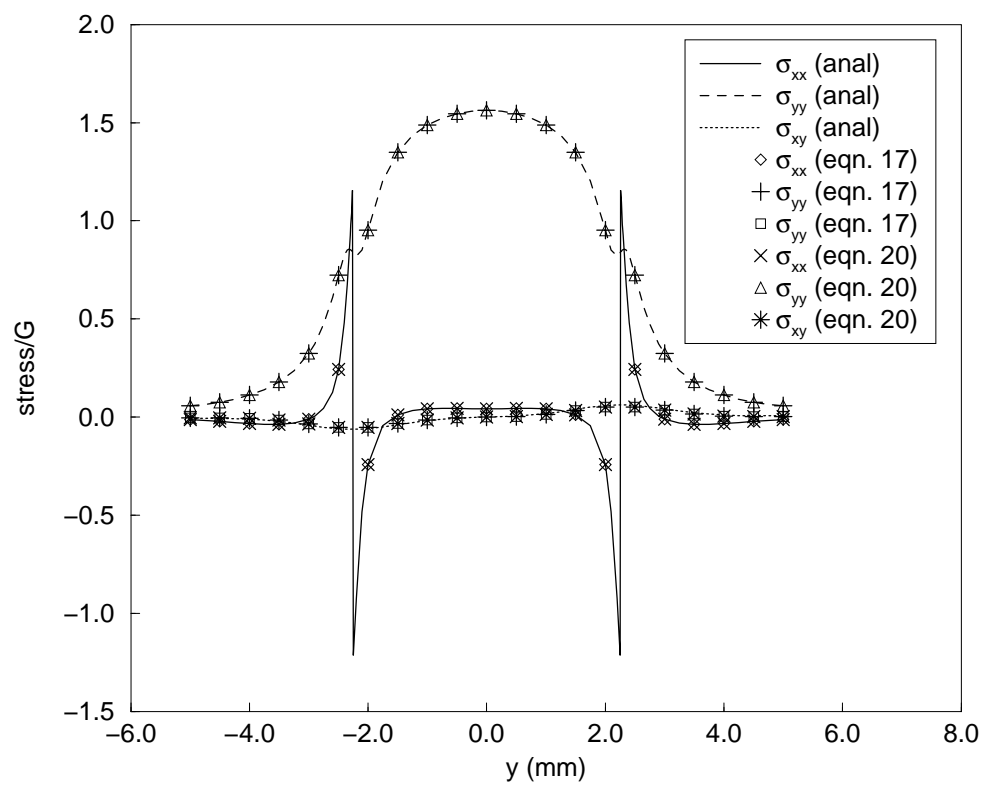

Fig. 4 Stresses produced by the inclusion of figure 2(b): comparison of analytical and numerical values.

\subsection{Discretization of the boundary-domain integral equations}

The loading boundary $\Gamma_{a}$ is discretized into straight quadratic boundary elements, totaling $N_{a}$ interpolation nodes. The coating $\Omega^{c}$ is modelled by $M$ rectangular cells $\Omega_{m}(1 \leq m \leq M)$, while the potentially plastic region of the substratum $\hat{\Omega}_{s}$, which is assumed to have a finite depth, is modelled by $N$ rectangular cells $\Omega_{n}$ $(1 \leq n \leq N)$. Both sets of cells include infinite cells, which are used to take into account the nonzero strains arising at infinity due to the fact that the loads are moving. In the present development, a piecewise constant interpolation is used for the unknown strains. Infinite cells in particular support the (unknown) limiting strain values at infinity in the horizontal direction. Eqs. (18) and (21) are collocated at the centers of all rectangular bounded cells; one collocation point is also chosen on each infinite cell. For each collocation point, the region $\Omega_{e}$ around $P$ is taken as the cell containing $P$; that together with the piecewise-constant strain interpolation implies that all integrals over $\Omega_{e}$ in Eqs. (18) and (21) vanish.

The discretization of Eq. (13) along these lines and for $P=P_{s} \in \Gamma_{a}\left(1 \leq s \leq N_{a}\right)$ leads to:

$$
\begin{aligned}
u_{k}\left(P_{s}\right)= & \sum_{r=1}^{N_{a}} t_{i}^{r} \int_{\Gamma_{a}} N_{r}(q) U_{i k}\left(P_{s}, q\right) \mathrm{d} \Gamma(q)+\sum_{1 \leq p \leq M} \hat{\varepsilon}_{a b}^{p} \int_{\Omega_{p}} L_{i j a b}^{c} E_{i j k}\left(P_{s}, Q\right) \mathrm{d} \Omega(Q) \\
& +\sum_{1 \leq p \leq M} \varepsilon_{a b}^{p} \int_{\Omega_{p}} \Delta L_{i j a b} E_{i j k}\left(P_{s}, Q\right) \mathrm{d} \Omega(Q)+\sum_{1 \leq q \leq N} \hat{\varepsilon}_{i j}^{q} \int_{\Omega_{q}} \Sigma_{i j k}\left(P_{s}, Q\right) \mathrm{d} \Omega(Q)
\end{aligned}
$$


where $N_{q}(q)$ denotes the interpolation function associated with the $q$-th boundary element node on $\Gamma_{a}$. Similarly, Eq. (18), the strain representation formula, becomes for $P=P_{m} \in \Omega^{c}$ :

$$
\begin{aligned}
\varepsilon_{k \ell}^{m} & -\varepsilon_{a b}^{m} \int_{\partial \Omega_{m}} \Delta L_{i j a b} n_{j}(q) U_{k \ell i}^{\star}\left(P_{m}, q\right) \mathrm{d} \Gamma(q)-\sum_{1 \leq p \leq M, p \neq m} \varepsilon_{a b}^{p} \int_{\Omega_{p}} \Delta L_{i j a b} E_{k \ell i j}^{\star}\left(P_{m}, Q\right) \mathrm{d} \Omega(Q) \\
& -\sum_{r=1}^{N_{a}} t_{i}^{r} \int_{\Gamma_{a}} N_{r}(q) U_{k \ell i}^{\star}\left(P_{m}, q\right) \mathrm{d} \Gamma(q)-\hat{\varepsilon}_{a b}^{m} \int_{\partial \Omega_{m}} L_{i j a b}^{c} n_{j}(q) U_{k \ell i}^{\star}\left(P_{m}, q\right) \mathrm{d} \Gamma(q) \\
& -\sum_{1 \leq p \leq M, p \neq m} \hat{\varepsilon}_{a b}^{p} \int_{\Omega_{p}} L_{i j a b}^{c} E_{k \ell i j}^{\star}\left(P_{m}, Q\right) \mathrm{d} \Omega-\sum_{1 \leq q \leq N} \hat{\varepsilon}_{i j}^{q} \int_{\Omega_{q}} \Sigma_{k \ell i j}^{\star}\left(P_{m}, Q\right) \mathrm{d} \Omega(Q)=0
\end{aligned}
$$

while (21), the strain representation formula at $P=P_{n} \in \Omega^{s}$, becomes:

$$
\begin{aligned}
\varepsilon_{k \ell}^{m} & -\sum_{1 \leq p \leq M} \varepsilon_{a b}^{p} \int_{\Omega_{p}} \Delta L_{i j a b} E_{k \ell i j}^{\star}\left(P_{n}, Q\right) \mathrm{d} \Omega(Q) \\
& -\sum_{q=1}^{N_{a}} t_{i}^{q} \int_{\Gamma_{a}} N_{q}(q) U_{k \ell i}^{\star}\left(P_{n}, q\right) \mathrm{d} \Gamma(q)-\hat{\varepsilon}_{a b}^{m} \int_{\partial \Omega_{n}} L_{i j a b} n_{j}(q) U_{k \ell i}^{\star}\left(P_{n}, q\right) \mathrm{d} \Gamma(q) \\
& -\sum_{1 \leq p \leq M} \hat{\varepsilon}_{a b}^{p} \int_{\Omega_{p}} L_{i j a b}^{c} E_{k \ell i j}^{\star}\left(P_{n}, Q\right) \mathrm{d} \Omega(Q)-\sum_{1 \leq q \leq N, q \neq n} \hat{\varepsilon}_{i j}^{q} \int_{\Omega_{q}} \Sigma_{k \ell i j}^{\star}\left(P_{n}, Q\right) \mathrm{d} \Omega(Q)=0
\end{aligned}
$$

Again, note that Eqs. (23) and (24) do not involve any singular integration, because the piecewiseconstant strain interpolation allowed one to recast all potentially singular integrals into (nonsingular) contour integrals over $\partial \Omega_{m}$ in Eq. (23) and over $\partial \Omega_{n}$ in Eq. (24).

For the numerical evaluation of integrals on cells which extend horizontally to infinity (or on their boundary), the following mapping is used for the horizontal coordinate $x_{2}(Q)$ :

$$
\pm x_{2}(Q)=r_{0} \frac{3+\xi}{1-\xi} \quad(-1 \leq \xi \leq+1)
$$

where the infinite element is such that either $r_{0} \leq x_{2}(Q) \leq+\infty$ or $-\infty \leq x_{2}(Q) \leq-r_{0}$, the \pm sign being adjusted accordingly. In particular, this mapping is such that:

$$
\frac{1}{|q-P|^{2}} d y(Q)=\left\{\frac{4}{r_{0}(3+\xi)^{2}}+o(|1-\xi|)\right\} d \xi
$$

Since all kernels in the domain cell integrations are $O\left(|q-P|^{2}\right)$, all integrals over infinite cells are thus converted into nonsingular integrals over a bounded region in the parameter space and thus can be evaluated using ordinary Gaussian quadrature.

Eqs. (23) and (24) have therefore the following respective matrix forms:

$$
\begin{aligned}
& \{\mathbf{0}\}=\left[\hat{\boldsymbol{B}}_{c c}\right]\left\{\hat{\boldsymbol{\varepsilon}}^{c}\right\}+\left[\hat{\boldsymbol{B}}_{c s}\right]\left\{\hat{\boldsymbol{\varepsilon}}^{s}\right\}+\left[\boldsymbol{B}_{c c}\right]\left\{\boldsymbol{\varepsilon}^{c}\right\}+\left\{\boldsymbol{f}_{c}\right\} \\
& \{\mathbf{0}\}=\left[\hat{\boldsymbol{B}}_{s c}\right]\left\{\hat{\boldsymbol{\varepsilon}}^{c}\right\}+\left[\hat{\boldsymbol{B}}_{s s}\right]\left\{\hat{\boldsymbol{\varepsilon}}^{s}\right\}+\left[\boldsymbol{B}_{s c}\right]\left\{\boldsymbol{\varepsilon}^{c}\right\}+\left\{\boldsymbol{\varepsilon}_{s}\right\}+\left\{\boldsymbol{f}_{s}\right\}
\end{aligned}
$$

where subscripts $c$ and $s$ refer to the coating and the substratum, respectively.

For a finite step, Eqs. (26) and (27) can, respectively, be replaced by the finite incremental forms

$$
\begin{aligned}
& \{\boldsymbol{0}\}=\left[\hat{\boldsymbol{B}}_{c c}\right]\left\{\Delta \hat{\boldsymbol{\varepsilon}}^{c}\right\}+\left[\hat{\boldsymbol{B}}_{c s}\right]\left\{\Delta \hat{\varepsilon}^{s}\right\}+\left[\boldsymbol{B}_{c c}\right]\left\{\Delta \varepsilon^{c}\right\}+\left\{\Delta \boldsymbol{f}_{c}\right\} \\
& \{\mathbf{0}\}=\left[\hat{\boldsymbol{B}}_{s c}\right]\left\{\hat{\boldsymbol{\varepsilon}}^{c}\right\}+\left[\hat{\boldsymbol{B}}_{s s}\right]\left\{\hat{\boldsymbol{\varepsilon}}^{s}\right\}+\left[\boldsymbol{B}_{s c}\right]\left\{\Delta \varepsilon^{c}\right\}+\left\{\Delta \boldsymbol{\varepsilon}_{s}\right\}+\left\{\Delta \boldsymbol{f}_{s}\right\}
\end{aligned}
$$




\subsection{Euler backward algorithm for isotropic material}

Euler backward method, for isotropic materials, acturally reduces to an elastic predictor-radial return method (Simo and Taylor, 1985) in the context of rate-independent plasticity with the Von Mises yield criterion and an associated flow rule. The yield criterion is, assuming linear isotropic hardening, is

$$
f(s) \equiv|s|-\sqrt{2 / 3}\left(k+E^{p} \bar{e}^{p}\right)=0
$$

where $s=\boldsymbol{\sigma}-\frac{1}{3} \operatorname{tr}(\boldsymbol{\sigma}) \mathbf{1}$ is the deviatoric stress (1: second-order unit tensor) and $|s|=(s: s)^{1 / 2}, \bar{e}^{p}$ is the cumulated equivalent plastic strain, $k$ is the shear yield strength and $E^{p}$ the hardening modulus.

A trial deviatoric stress is introduced as

$$
\boldsymbol{s}_{n+1}^{T}=\boldsymbol{s}_{n}+2 G \Delta \boldsymbol{e}_{n}
$$

where $e$ is the deviatoric strain. The deviatoric stress $s_{n+1}$ at the end of the step is then given by

$$
\boldsymbol{s}_{n+1}=\boldsymbol{s}_{n+1}^{T}-2\left(G+E^{p} / 3\right) \lambda \boldsymbol{n}
$$

where

$$
\begin{gathered}
n=\boldsymbol{s}_{n+1} /\left|\boldsymbol{s}_{n+1}\right| \\
\bar{e}_{n+1}^{p}-\bar{e}_{n}^{p}=\sqrt{2 / 3} \lambda
\end{gathered}
$$

Due to $\boldsymbol{s}_{n+1}=\left|\boldsymbol{s}_{n+1}\right| \boldsymbol{n}, \boldsymbol{n}$ is also determined in terms of the trial elastic stress $\boldsymbol{s}_{n+1}^{T}$ according to

$$
\boldsymbol{n}=\boldsymbol{s}_{n+1}^{T} /\left|\boldsymbol{s}_{n+1}^{T}\right|
$$

From (31) and (32), it then follows that the enforcement of the consistency condition reduces to a simple scalar equation, which yields:

$$
\lambda=\frac{\left|s_{n+1}^{T}\right|-\sqrt{2 / 3}\left(k+E^{p} \bar{e}_{n}^{p}\right)}{\left(2 G+2 E^{p} / 3\right)}
$$

As a result, the plastic strain increment induced by a given total strain increment $\Delta \varepsilon$ is:

$$
\Delta \hat{\varepsilon}=\lambda \boldsymbol{n}
$$

Besides, one can define a local tangent operator $\boldsymbol{D}$ through:

$$
\frac{\partial \Delta \hat{\varepsilon}}{\partial \Delta \varepsilon}=\boldsymbol{D}\left(\Delta \varepsilon ; \mathcal{S}_{0}\right)
$$

where $\mathcal{S}_{0}$ symbolizes the values of the mechanical variables before application of the strain increment (i.e. for $\Delta \varepsilon=0$ ). The tangent operator $D$, obtained by differentiating Eq. (35) with respect to $\Delta \varepsilon$, is given by:

$$
\boldsymbol{D}\left(\Delta \varepsilon ; \mathcal{S}_{0}\right)=\frac{1}{\left|\boldsymbol{s}_{n+1}^{T}\right|}\left[\frac{3 G}{3 G+E^{p}} \sqrt{2 / 3}\left(k+E^{p} \bar{e}_{n}^{p}\right) \boldsymbol{n} \otimes \boldsymbol{n}+2 G \lambda\left(\boldsymbol{I}-\frac{1}{3} \mathbf{1} \otimes \mathbf{1}\right)\right]
$$

An extension of this very common constitutive integration scheme to anisotropic plasticity is proposed in De Borst and Feenstra (1990). 


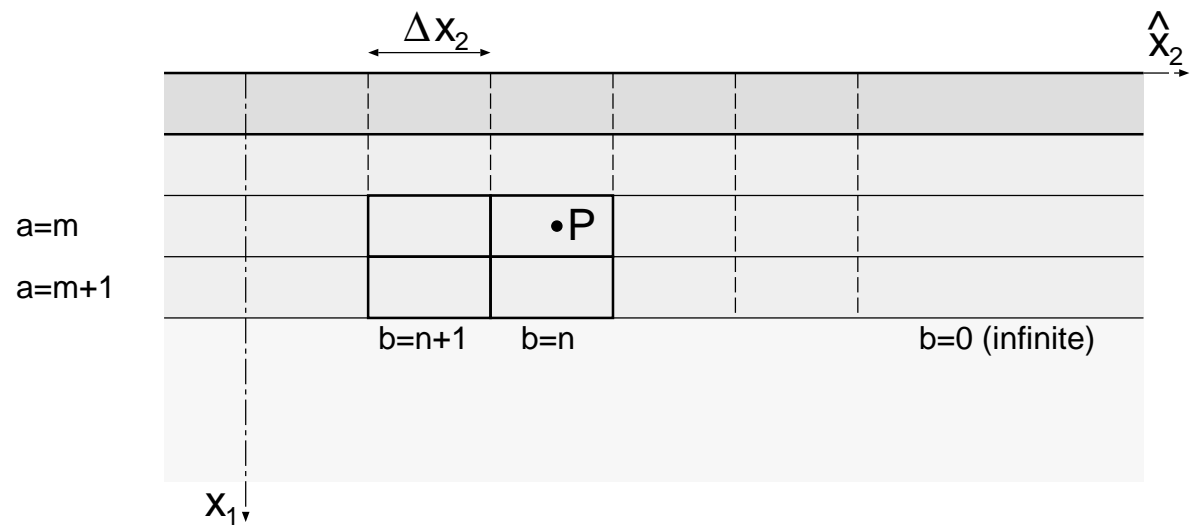

Fig. 5 Discretization into integration cells

\subsection{Elastoplastic steady-state algorithm}

The elastoplastic solution algorithm follows closely the adaptation of the Simo and Taylor (1985) approach proposed in Bonnet and Mukherjee (1996): equations (28)-(29) are solved for the unknown increments of total and plastic strains, using the consistent tangent operator.

In the steady-state case, the computational domain is horizontally infinite, and the infinite cells are used. Collocation points with the same $x_{1}$ coordinate are numbered using two indices $a, b$ which range in the vertical and horizontal subdivisions of the rectangular mesh, respectively. The index $b$ takes increasing consecutive values in the direction opposite to the motion (figure 5), and in particular the rightmost infinite cells are labelled by $b=0$; this arrangement is similar to that made in Dang Van and Maitournam (1993) in the context of finite element method, except that no infinite cells were used in the latter reference. The horizontal width $\Delta x_{2}$ of the cells is related to the time step through

$$
\Delta t=\Delta x_{2} / V
$$

and in particular must therefore be constant over the mesh. As a consequence, the strain increment $\Delta \varepsilon_{a, b}$ becomes a differences between this cell and its left horizontal neighbour:

$$
\{\Delta \varepsilon\}_{b}=\{\varepsilon\}_{b+1}-\{\varepsilon\}_{b}
$$

(where the 'vector' $\{\varepsilon\}_{b}$ gathers the values of $\varepsilon_{a, b}$ for a fixed index $b$ ) and similarly for the increments of plastic strains. Also, the initial distribution of plastic strain $\{\hat{\varepsilon}\}^{I}$ is prescribed through:

$$
\{\hat{\varepsilon}\}_{b=0}=\{\hat{\varepsilon}\}^{I}
$$


To solve globally for the plastic and total strain increments, the Newton method is applied to the system (28)-(29). The additive corrections $\Delta \varepsilon_{a, b}^{(i+1)}=\Delta \varepsilon_{a, b}^{(i)}+\delta \varepsilon_{a, b}^{(i)}$ thus solve the linear system of equations

$$
\begin{gathered}
{\left[\hat{\boldsymbol{B}}_{c c}\right]\left\{\boldsymbol{D}^{c}: \delta \boldsymbol{\varepsilon}^{c(i)}\right\}+\left[\hat{\boldsymbol{B}}_{c s}\right]\left\{\boldsymbol{D}^{s}: \delta \hat{\boldsymbol{\varepsilon}}^{s(i)}\right\}+\left[\boldsymbol{B}_{c c}\right]\left\{\delta \varepsilon^{c(i)}\right\}} \\
=-\left[\hat{\boldsymbol{B}}_{c c}\right]\left\{\Delta \hat{\boldsymbol{\varepsilon}}^{c}\right\}-\left[\hat{\boldsymbol{B}}_{c s}\right]\left\{\Delta \hat{\boldsymbol{\varepsilon}}^{s}\right\}-\left[\boldsymbol{B}_{c c}\right]\left\{\Delta \boldsymbol{\varepsilon}^{c}\right\}-\left\{\Delta \boldsymbol{f}_{c}\right\} \\
{\left[\hat{\boldsymbol{B}}_{s c}\right]\left\{\boldsymbol{D}^{c}: \delta \boldsymbol{\varepsilon}^{c(i)}\right\}+\left[\hat{\boldsymbol{B}}_{s s}\right]\left\{\boldsymbol{D}^{s}: \delta \boldsymbol{\varepsilon}^{s(i)}\right\}+\left[\boldsymbol{B}_{s c}\right]\left\{\delta \boldsymbol{\varepsilon}^{c(i)}\right\}+\left\{\delta \boldsymbol{\varepsilon}_{s(i)}\right\}} \\
=-\left[\hat{\boldsymbol{B}}_{s c}\right]\left\{\hat{\boldsymbol{\varepsilon}}^{c}\right\}-\left[\hat{\boldsymbol{B}}_{s s}\right]\left\{\hat{\boldsymbol{\varepsilon}}^{s}\right\}-\left[\boldsymbol{B}_{s c}\right]\left\{\Delta \boldsymbol{\varepsilon}^{c}\right\}-\left\{\Delta \boldsymbol{\varepsilon}_{s}\right\}-\left\{\Delta \boldsymbol{f}_{s}\right\}
\end{gathered}
$$

and the iterates $\left\{\delta \varepsilon^{(i)}\right\}$ are computed until the system (28)-(29) is satisfied.

The solution algorithm for the steady-state case is:

(1) initialization: $\left\{\hat{\boldsymbol{\varepsilon}}^{s(i=0)}\right\}_{b}=\{\hat{\boldsymbol{\varepsilon}}\}^{I}(b=0,1, \ldots)$;

(2) Calculation of $\left\{\varepsilon^{c(i)}\right\}$ and $\left\{\varepsilon^{s(i)}\right\}$ from Eqs. (28) and (29), respectively;

(3) Calculation of $\left\{\boldsymbol{s}^{(i)}\right\}:\left\{\boldsymbol{s}^{s(i)}\right\}=\left\{\boldsymbol{L}:\left(\boldsymbol{e}^{s(i)}-\hat{\boldsymbol{\varepsilon}}^{s(i)}\right)\right\}$ for the substratum

if $f\left(\left\{\boldsymbol{s}^{s(i)}\right\}\right) \leq\{\mathbf{0}\}$ then

goto (5)

else

$$
\begin{aligned}
& \text { for } b=0,1, \ldots \text { do } \\
& \left\{\boldsymbol{s}^{s(i+1)}\right\}_{b+1}^{T}=\left\{\boldsymbol{s}^{s(i)}\right\}_{b}+2 G\left\{\Delta \boldsymbol{e}^{s(i)}\right\}(b=0,1, \ldots) \\
& \text { if } f\left(\left\{\boldsymbol{s}^{s(i+1)}\right\}_{b+1}^{T}\right)>\{\mathbf{0}\} \text { then } \\
& \{\boldsymbol{n}\}_{b+1}=\left\{\frac{\boldsymbol{s}^{s(i+1) T}}{\left|\boldsymbol{s}^{s(i+1) T}\right|}\right\}_{b+1} ;\{\lambda\}_{b+1}=\left\{\frac{\left|\boldsymbol{s}^{s(i+1) T}-\sqrt{2 / 3} k\right|}{2 G+2 / 3 E^{p}}\right\}_{b+1} ; \\
& \quad\left\{\hat{\boldsymbol{\varepsilon}}^{s(i+1)}\right\}_{b+1}=\left\{\hat{\boldsymbol{\varepsilon}}^{s(i+1)}\right\}_{b}+\{\lambda \boldsymbol{n}\}_{b+1} ;
\end{aligned}
$$

else

$$
\left\{\boldsymbol{s}^{s(i+1)}\right\}_{b+1}=\left\{\boldsymbol{s}^{s(i+1)}\right\}_{b+1}^{T}
$$

end if

end for

end if

(4) Prepare for next iteration: $i:=i+1$, goto (2);

(5) end.

\subsection{Elastic contact stress analysis}

The elastic roller (Figure 6), ocupying the domain $\Omega^{r}$ of boundary $\Gamma^{r}$ is modelled by means of ordinary displacement boundary integral equations, using the Kelvin (full-space) fundamental solution. Introducing a boundary element discretization and collocating the resulting integral equation at displacement nodes yields the relation:

$$
[\boldsymbol{H}]\{\boldsymbol{u}\}=[\boldsymbol{G}]\{\boldsymbol{t}\}
$$




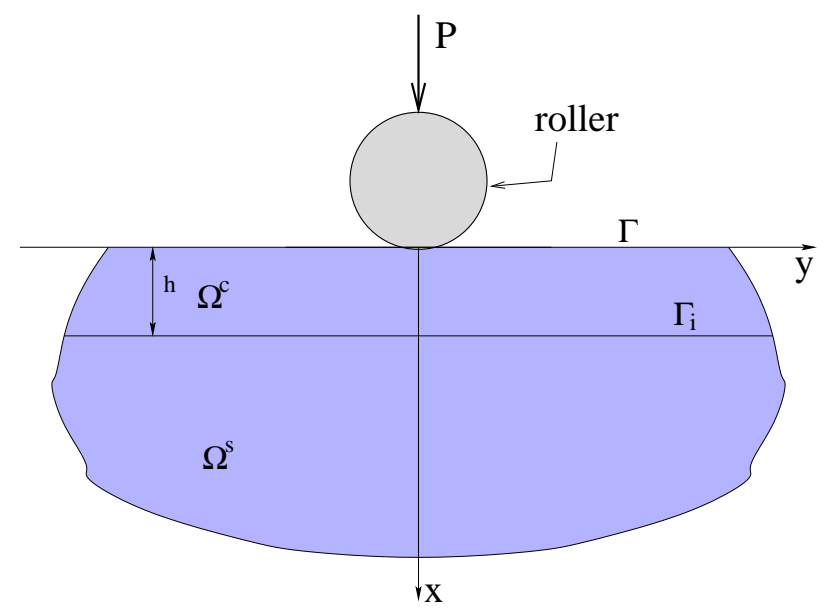

Fig. 6 A roller over a coated half-space

where $\{\boldsymbol{u}\}$ and $\{\boldsymbol{t}\}$ are vectors of nodal displacements and tractions on the roller boundary and $[\boldsymbol{H}]$ and $[\boldsymbol{G}]$ are the BEM coefficient matrices. After introducing boundary conditions away from the area of potential contact $\Gamma_{a}^{r}$, the system (40) is reduced by condensation into a system of equations for the displacements $\left\{\boldsymbol{u}_{a}^{r}\right\}$ and tractions $\left\{\boldsymbol{t}_{a}^{r}\right\}$ on $\Gamma_{a}^{r}$ :

$$
\left[\boldsymbol{H}_{a a}^{r}\right]\left\{\boldsymbol{u}_{a}^{r}\right\}=\left[\boldsymbol{G}_{a a}^{r}\right]\left\{\boldsymbol{t}_{a}^{r}\right\}+\left\{\boldsymbol{f}_{a}^{r}\right\}
$$

where the superscript $r$ indicates quantities defined on the roller, $\left[\boldsymbol{H}_{a a}^{r}\right]$ and $\left[\boldsymbol{G}_{a a}^{r}\right]$ are the condensed BEM coefficent matrices and the vector $\left\{\boldsymbol{f}_{a}^{r}\right\}$ incorporates the contribution of known boundary data.

Assuming that both the coating and the substratum remain elastic, the discretized equations (22) and (26) reduce to:

$$
\begin{aligned}
\left\{\boldsymbol{u}_{a}\right\} & =\left[\boldsymbol{G}_{a a}\right]\left\{\boldsymbol{t}_{a}\right\}+\left[\boldsymbol{C}_{a c}\right]\left\{\boldsymbol{\varepsilon}^{c}\right\} \\
{\left[\boldsymbol{B}_{c c}\right]\left\{\boldsymbol{\varepsilon}^{c}\right\} } & =\left[\boldsymbol{D}_{c a}\right]\left\{\boldsymbol{t}_{a}\right\}
\end{aligned}
$$

The displacements $\left\{\boldsymbol{u}_{a}\right\}$ can be expressed in terms of contact tractions $\left\{\boldsymbol{t}_{a}\right\}$ by eliminating $\left\{\varepsilon^{c}\right\}$ between the two equations; one obtains:

$$
\left\{\boldsymbol{u}_{a}\right\}=\left[\boldsymbol{R}_{1}\right]\left\{\boldsymbol{t}_{a}\right\} \quad \text { with } \quad\left[\boldsymbol{R}_{1}\right]=\left[\boldsymbol{C}_{a c}\right]\left[\boldsymbol{B}_{c c}\right]^{-1}\left[\boldsymbol{D}_{c a}\right]+\left[\boldsymbol{G}_{a a}\right]
$$

On the area of potential contact, we introduce the following equilibrium and compatibility conditions

$$
\begin{gathered}
\left\{\boldsymbol{u}_{a}\right\}-\left\{\boldsymbol{u}_{a}^{r}\right\}=\{\boldsymbol{\Delta}\}-\{\boldsymbol{U}\} \\
\left\{\boldsymbol{t}_{a}\right\}+\left\{\boldsymbol{t}_{a}^{r}\right\}=\{\boldsymbol{0}\}
\end{gathered}
$$

where $\{\boldsymbol{U}\}$ stands for relative position vector between contact points pairs and $\{\boldsymbol{\Delta}\}$ is the initial gap vector of the relative contact point pairs. Using these two equations together with Eqs. (41) and (42), the following 
matrix equation between the relative displacement $\{\boldsymbol{U}\}$ and the traction $\left\{\boldsymbol{t}_{a}\right\}$ on $\Gamma_{a}$ is obtained:

$$
\left[\boldsymbol{R}_{2}\right]\left\{\boldsymbol{t}_{a}\right\}-\left[\boldsymbol{H}_{a a}^{r}\right]\{\boldsymbol{U}\}=\{\boldsymbol{F}\}
$$

where

$$
\begin{aligned}
& {\left[\boldsymbol{R}_{2}\right]=\left[\boldsymbol{H}_{a a}^{r}\right]\left[\boldsymbol{R}_{1}\right]+\left[\boldsymbol{G}_{a a}^{r}\right]} \\
& \{\boldsymbol{F}\}=\left[\boldsymbol{H}_{a a}^{r}\right]\{\boldsymbol{\Delta}\}-\left\{\boldsymbol{f}_{a}^{r}\right\}
\end{aligned}
$$

To solve the contact problem, Eq. (45) is supplemented with the Coulomb friction law and the nonpenetration condition $\left\{\boldsymbol{U}_{n}\right\} \geq\{\mathbf{0}\}$. The contact solution $\left\{\boldsymbol{t}_{a}\right\},\{\boldsymbol{U}\}$ is then found by means of an iterative procedure (see, for example, Antes, Steinfeld, and Tröndle (1991), Huesmann and Kuhn (1995) and many others).

\section{Numerical examples}

In order to test numerically the proposed boundary-domain integral equation for a coated half-space in 2-D plane strain, stress analysis in elastic contact state and elastoplastic implementations under statically Hertz contact and steady-state elastoplastic rolling contact have been studied.

\subsection{Example 1: elastic coated half-space indented by an elastic punch}

The indentation of a coated half-space by an elastic punch, in 2-D plane strain conditions, is considered, with the material and geometrical data as follows: $E($ punch $)=E_{s}($ substratum $)=210 \mathrm{GPa}, \nu($ punch $)=\nu_{c}$ $($ coating $)=\nu_{s}($ substratum $)=0.3$, punch radius $R=1 \mathrm{~m}$, total applied vertical load $P=2.2225 \times 10^{8} \mathrm{~N} / \mathrm{m}$ (see figure 7 , where the distributed load is $p=P /(2 R)$ ). The punch is not moving. Various values will

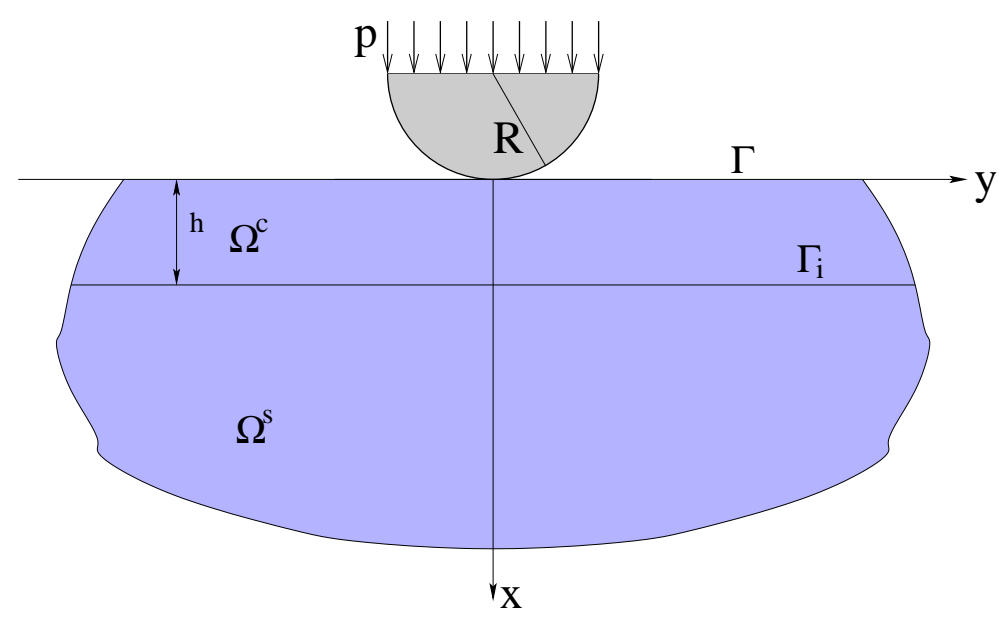

Fig. 7 Indentation of a coated half-space by an elastic punch 


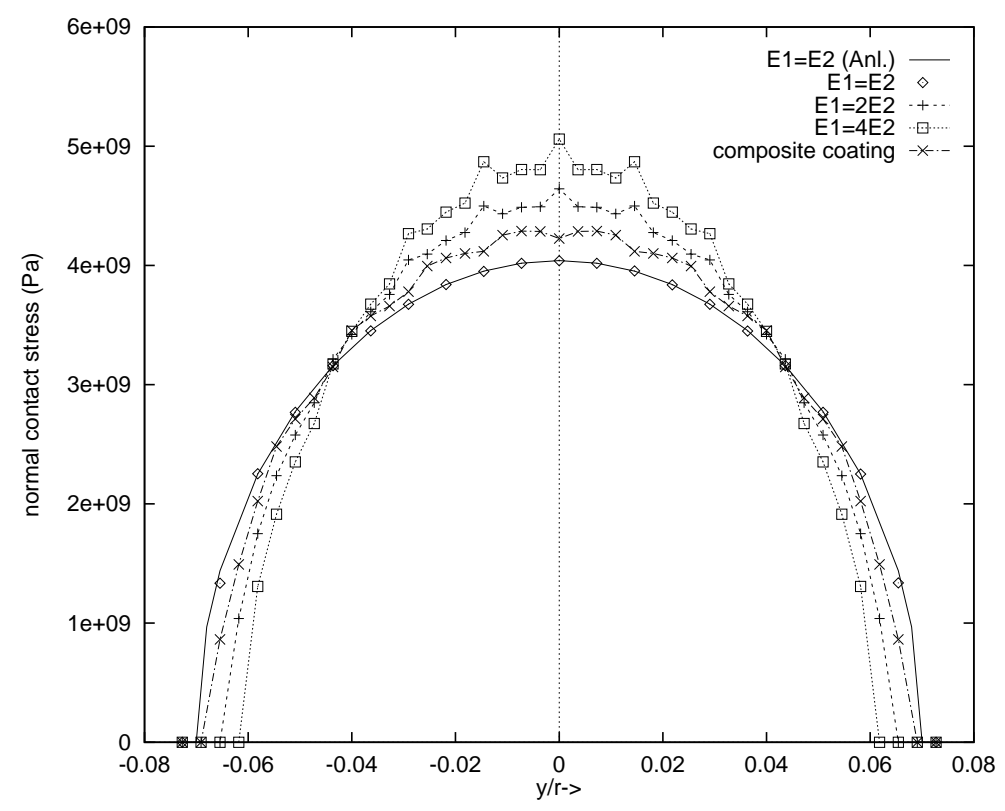

Fig. 8 Example 1: effect of the coating stiffness on normal contact stress

be considered for the thickness $h$, the Coulomb friction coefficient $\mu$ and the Young moduli of the coating. The punch is modelled using 62 quadratic isoparametric boundary elements, including 20 on the potential contact zone. The potential contact boundary on the coating is divided into 20 quadratic isoparametric elements. The coating is divided into 28 rectangular cells, including 2 infinite cells, for $h=0.1 \mathrm{~m}$.

For $h=0.1 \mathrm{~m}$ and frictionless contact $(\mu=0.0)$, normal contact stress distributions are shown in Figure 8 for several values of the coating stiffness $E_{c}$. For $E_{c}=E_{s}$, the present results agree well with

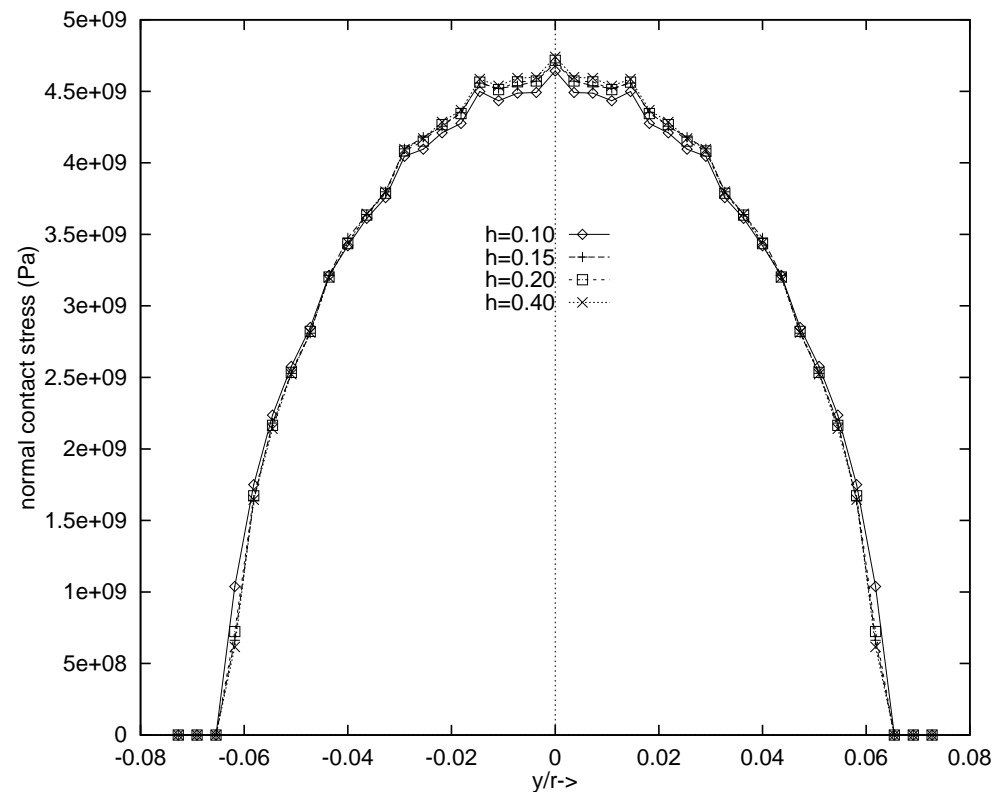

Fig. 9 Example 1: effect of the coating thickness on the normal contact stress 


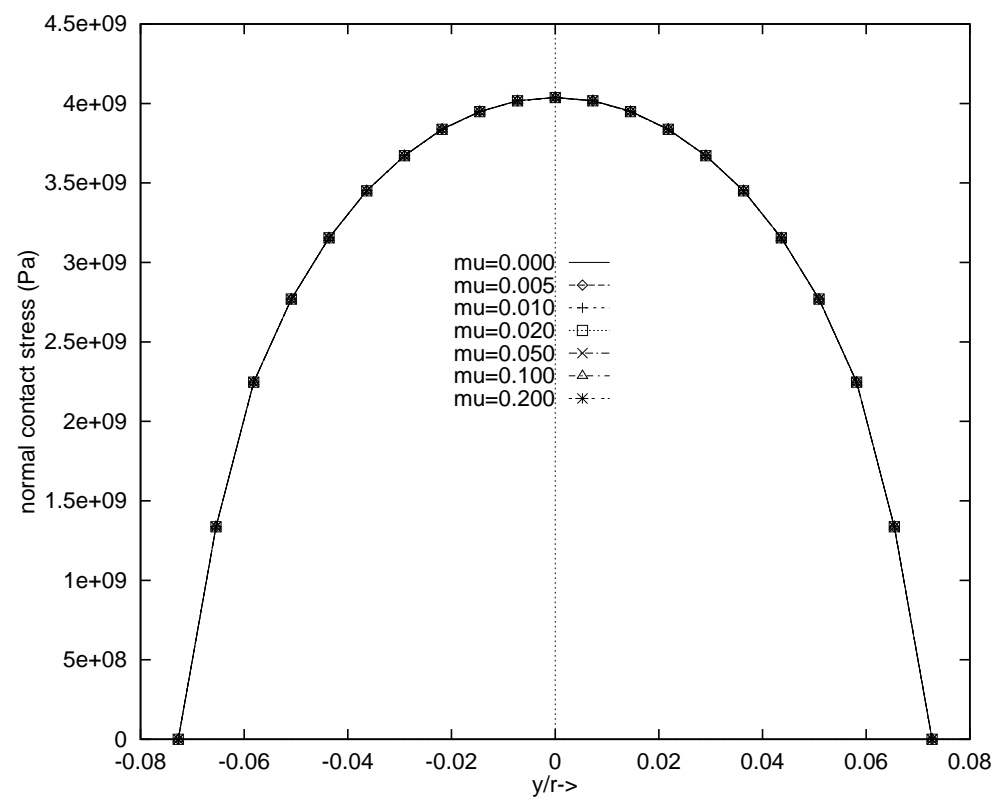

Fig. 10 Example 1: effect of the friction coefficient on the normal contact stress

the analytical solution. The maximum contact pressure is seen to increase with $E_{c}$, while the contact area decreases. Figure 8 also shows the normal contact stress distribution obtained for an anisotropic coating material with the following material constants: $E_{x}=570 \mathrm{GPa}, E_{y}=E_{z}=140 \mathrm{GPa}, G_{x y}=57 \mathrm{GPa}, \nu_{y x}=$ $0.068, \nu_{y z}=0.4$, and $\nu_{x z}=0.277$.

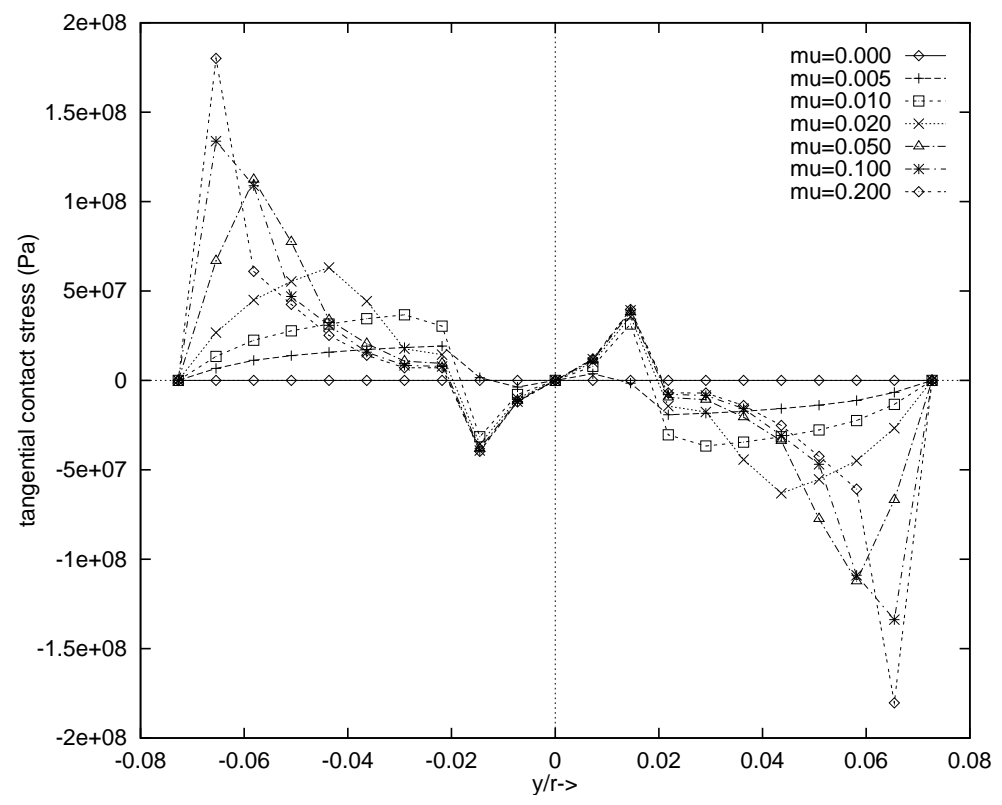

Fig. 11 Example 1: effect of the friction coefficient on the tangential contact stress 


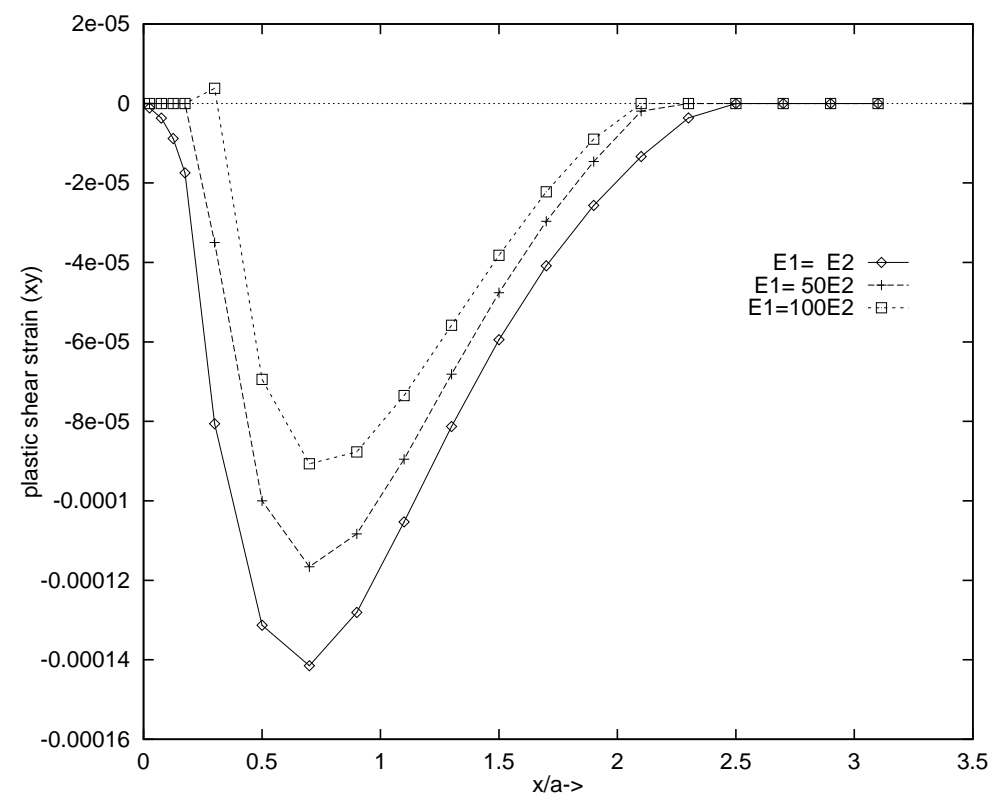

Fig. 12 Example 2: effect of the coating stiffness on plastic shear strain (plotted against $x$ for $y=0.1 a$ ), with $h=0.2 a$.

Next, assuming $E_{c}=2 E_{s}$, results for various coating thicknesses (meshes along the $y$-direction kept unchanged, and divisions along the $x$-direction are $1(h=0.1 \mathrm{~m}), 2(h=0.15 \mathrm{~m}), 2(h=0.2 \mathrm{~m})$ and 4 $(h=0.4 \mathrm{~m}))$ are shown in figure 9. The coating thickness is seen to have little effect on the results.

The influence of frictional coefficient on normal contact pressure and tangential contact traction is displayed in figure 10 and figure 11, respectively (with $E_{c}=E_{s}$ ). The tangential contact stress is seen to be much more influenced by the frictional coefficient than the normal contact stress. The stick area increases with the frictional coefficient.

\subsection{Example 2: elastic-plastic response of a coated half-space under a fixed Hertz load}

Here, the influence of the coating material parameters, the coating thickness and the frictional coefficient on the distribution of plastic strains in the substratum is considered. The elastic modulus of substratum is $E_{s}=$ $210 \mathrm{GPa}$, Poisson ratio $\nu_{c}=\nu_{s}=0.3$. The shear yield limit in the substratum is $\sigma_{k}=159.0 \mathrm{MPa}$; the Hertz maximum contact pressure is chosen as $P / k=4.5$, and the contact half-width as $a=1 \mathrm{~mm}$. The coating has been subdivided into $41(h=0.05 a), 82(h=0.1 a), 123(h=0.15 a)$ and $164(h=0.2 a)$ quadrilateral elements, two, two, eight and eight of which are infinite elements and the remaining are constant elements with size $0.05 a \times 0.2 a(\mathrm{a}=1 \mathrm{~mm}$ is the contact half-width). 315 elements were used in the substratum: 30 infinite elements and 285 quadrilateral constant elements of size $0.2 a \times 0.2 a$.

Figure 12 illustrates the influence of the coating Young modulus (with $h=0.2 a$ ): the plastic strains $\hat{\varepsilon}_{x y}$, plotted against $x$ for $y=0.1 a$, are seen to decrease with increasing stiffness of the coating, as expected. Similarly, figure (13) shows the decrease of plastic strains for a hard coating $\left(E_{c}=100 E_{s}\right)$ as the coating 
thickness increases. In both cases, frictionless contact (i.e. $\mu=0$ ) is assumed. Next, the influence of $\mu$ is shown (with $E_{c}=100 E_{s}, h=0.1 a$ ) in figure 14: plastic strains in the substratum are seen to increase with $\mu$. Finally, an anisotropic coating is considered, with the material parameters $E_{x}=10 E_{s}, E_{y}=E_{z}=E_{s}$, $\nu_{x y}=\nu_{x z}=0.3333, \nu_{y z}=0.3, \bar{\sigma}=115.118 \mathrm{MPa}, \alpha_{12}=\alpha_{31}=0.25, \alpha_{44}=0.3333, \alpha_{23}=1.75$ (Borst and Feenstra, 1990) and with $h=0.2 a$. Plastic strains at $y=0.2 a$ and along the $x$-direction are shown in figure 15 for two meshes characterized by subdivision parameters $M_{x}=4$ and $M_{x}=8$ respectively; results for both meshes are very similar.

\subsection{Example 3: elastic-plastic response of a coated half-space under a moving Hertz load}

The third example is used to investigate elastoplastic steady-state rolling contact, with constitutive and loading parameters as follows: Young modulus $E_{s}=207 \mathrm{GPa}$, Poisson ratio $\nu_{s}=\nu_{c}=0.3$, shear yield limit $k_{s}=159.118 \mathrm{MPa}$, hardening modulus $E_{s}^{p}=69 \mathrm{GPa}$. Hertz loading is assumed in this analysis, with a maximum Hertz contact pressure $P=5.0 k_{s}$. Various values are considered for the Young moduli in the coating and the friction coefficient between the roller and the coating. Meshes with 1818 elements (substratum), 36 of which are infinite elements (mesh 1) or with 738 elements, 36 of which are infinite elements (mesh 2), and 101 elements (coating), 4 of which are infinite element (mesh 1) or 41 elements, 2 of which are infinite elements (mesh 2$)$, are used. The cell size is $0.2 \mathrm{a}^{*} 0.2 \mathrm{a}(\mathrm{a}=0.5 \mathrm{~mm}$ is the contact half-width). The coating thickness is first assumed as $h=0.2 a$.

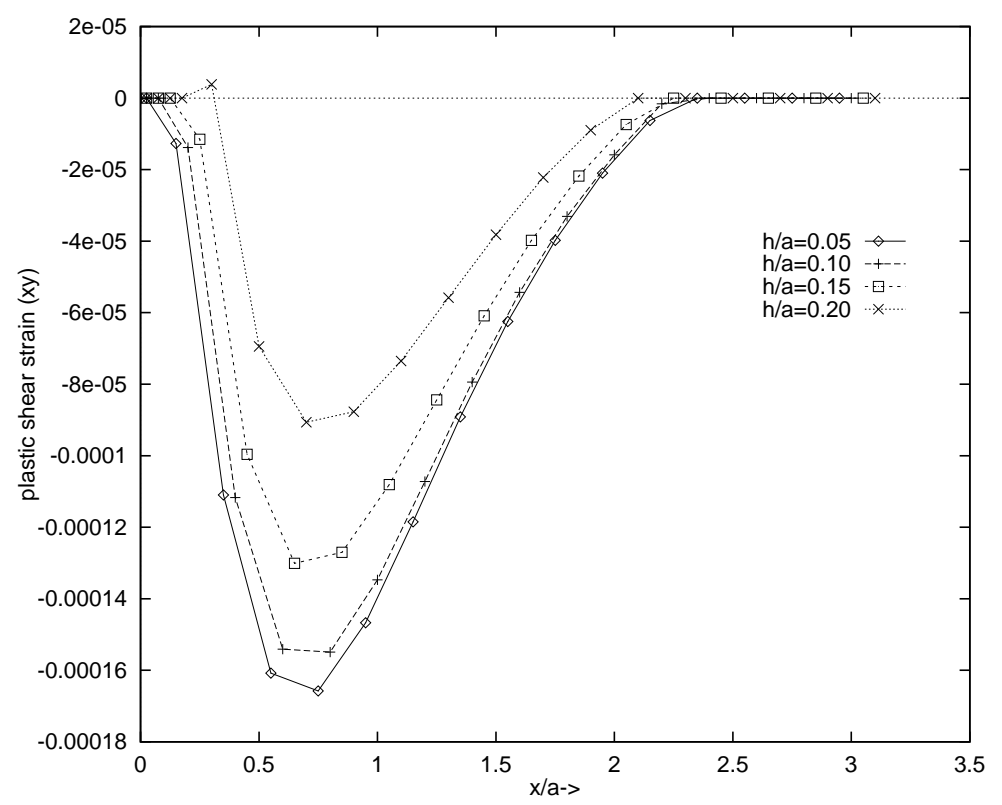

Fig. 13 Example 2: effect of the coating thickness on plastic shear strain (plotted against $x$ for $y=0.1 a$ ), with $E_{c}=100 E_{s}$ 


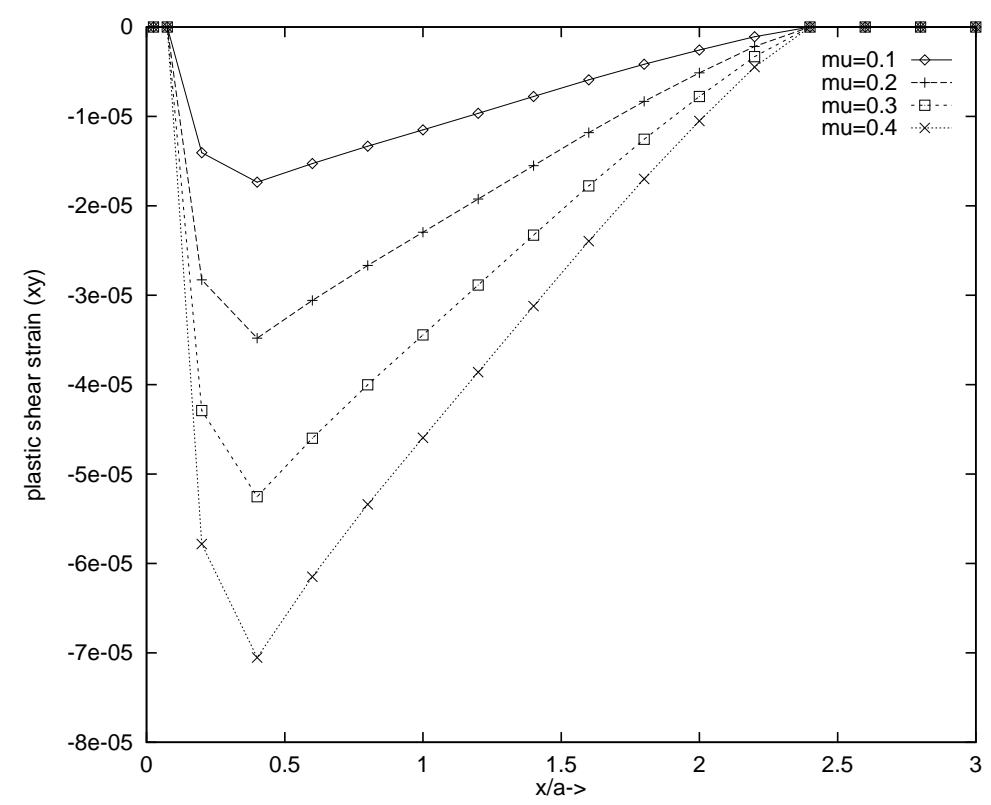

Fig. 14 Example 2: effect of the frictional coefficient on plastic shear strain $(y=0.0)$

The special case of a homogeneous half-space (i.e. coating and substratum have same material parameters) allows comparisons to other published results. Figure 16 displays the plastic stress $\sigma_{y y}$ against $x$ and under the load. The results obtained using the present approach are larger than those of Bhargava, Hahn, and Rubin (1988) and Dang Van and Maitournam (1993) obtained using the finite element method (FEM), but reproduce well those of Lederer, Bonnet, and Maitournam (1998) who also used a boundary-domain integral equation approach. The results obtained using meshes 1 and 2 are nearly identical, and the coarser mesh 2 will be used in the sequel. In addition, the results obtained for elastic shakedown $(x=1.15 a, y=0.0)$

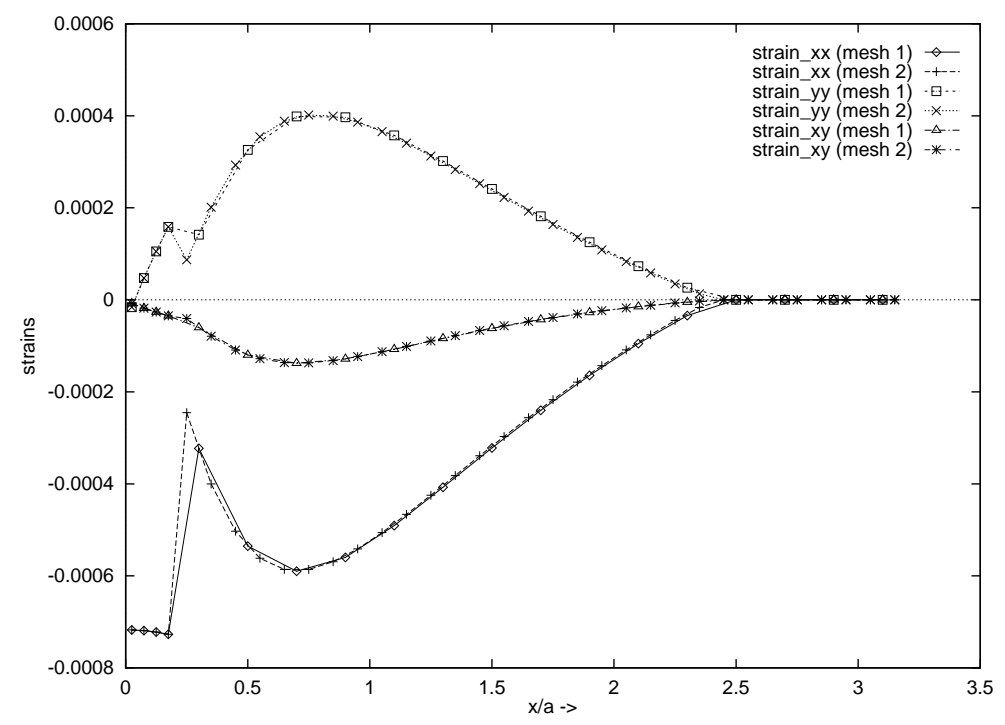

Fig. 15 Example 2: plastic distribution along the $x$-direction $(y=0.1 a)$ for $P / k=4.5$ and $\mu=0.0$ 


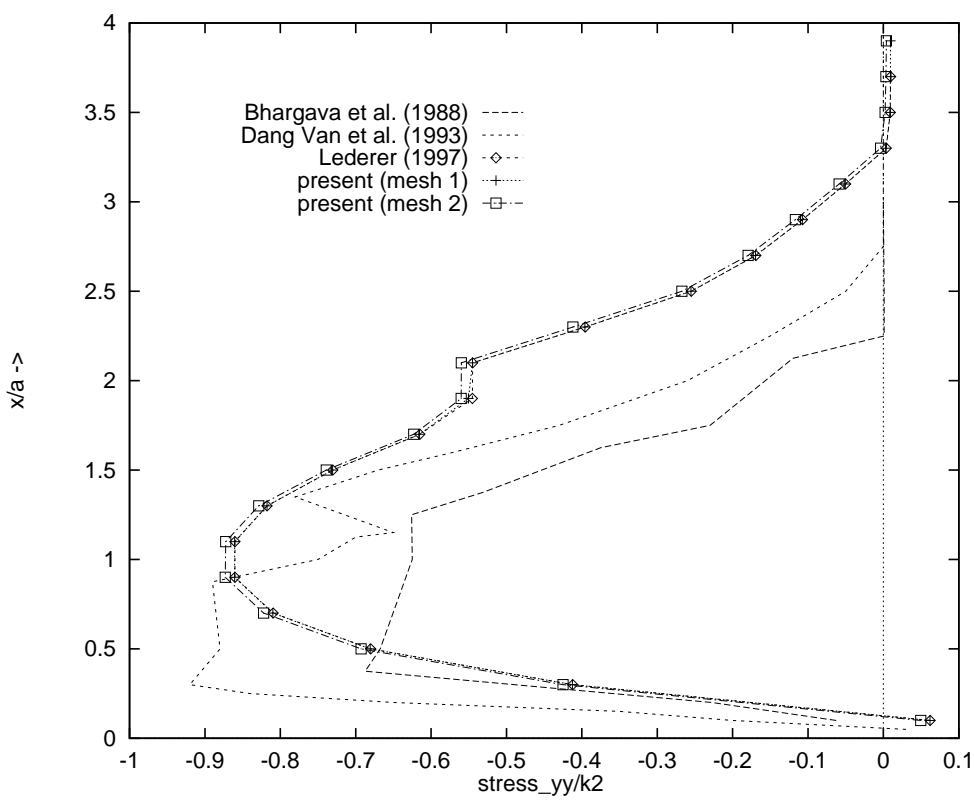

Fig. 16 Example 3: stress $\sigma y y$ along the $x$-axis

and plastic shakedown $(x=0.85 a, y=0.0)$ shown in Figs. 19 and 20, respectively, reproduce very well those of Lederer, Bonnet, and Maitournam (1998).

In an attempt to explain this discrepancy between FEM and BEM, the stress $\sigma_{y y}$ generated along the $x$-axis by constant initial strains $\left(\hat{\varepsilon}_{x x}=1\right.$., $\left.\hat{\varepsilon}_{y y}=-0.5, \hat{\varepsilon}_{x y}=0.0\right)$ on two symmetrical infinite inclusions defined by $\ell \leq x \leq 2 \ell$ and $r \ell \leq|y| \leq+\infty$ (see Fig. 17, where $\ell=1$ ) have been calculated. The results for $\sigma_{y y}(x, y=0)$ shown in Fig. 18 for various values of $r$ show that the stress created by constant plastic inclusions going to infinity is significant unless $r$ is quite large, i.e. the inclusions are quite remote. Here and in Lederer, Bonnet, and Maitournam (1998), the introduction of infinite cells allow to account properly for the possibility of nonzero (and asymptotically constant) plastic strain at infinity, which is seen here to have a significant impact on the overall results, whereas this is not the case in Bhargava, Hahn, and Rubin (1988) and Dang Van and Maitournam (1993).

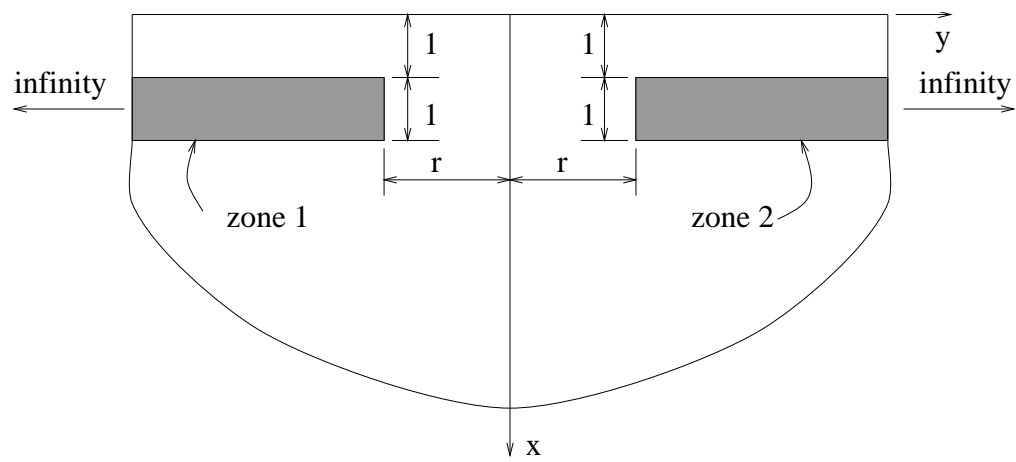

Fig. 17 Example 3: constant plastic strain zones in half-plane 


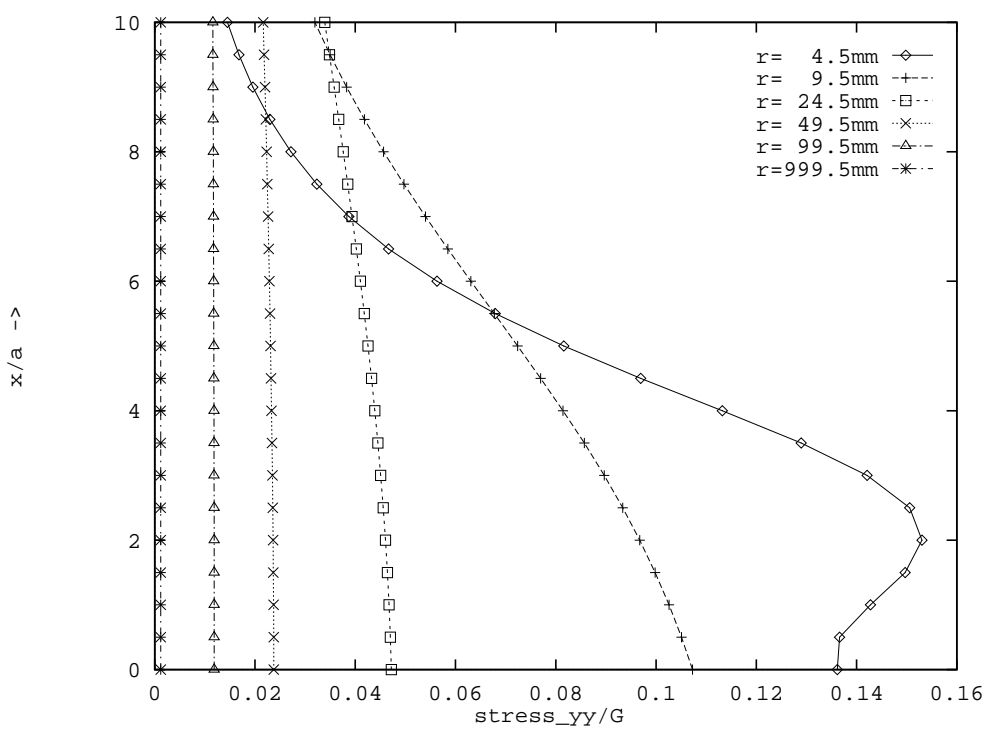

Fig. 18 Example 3: stress $\sigma y y$ along the $x$-axis from two unlimited constant plastic strains

Fig. 21 indicates that plastic shear strains decrease as the coating stiffness increases. The influence of friction coefficient and of coating thickness (for $E 1=50 E 2$, i.e. a very hard coating) on plastic shear strains are presented on Figs. 22 and 23, respectively; plastic shear strain is seen to increase with the friction coefficient and to decrease as the coating thickness increases.

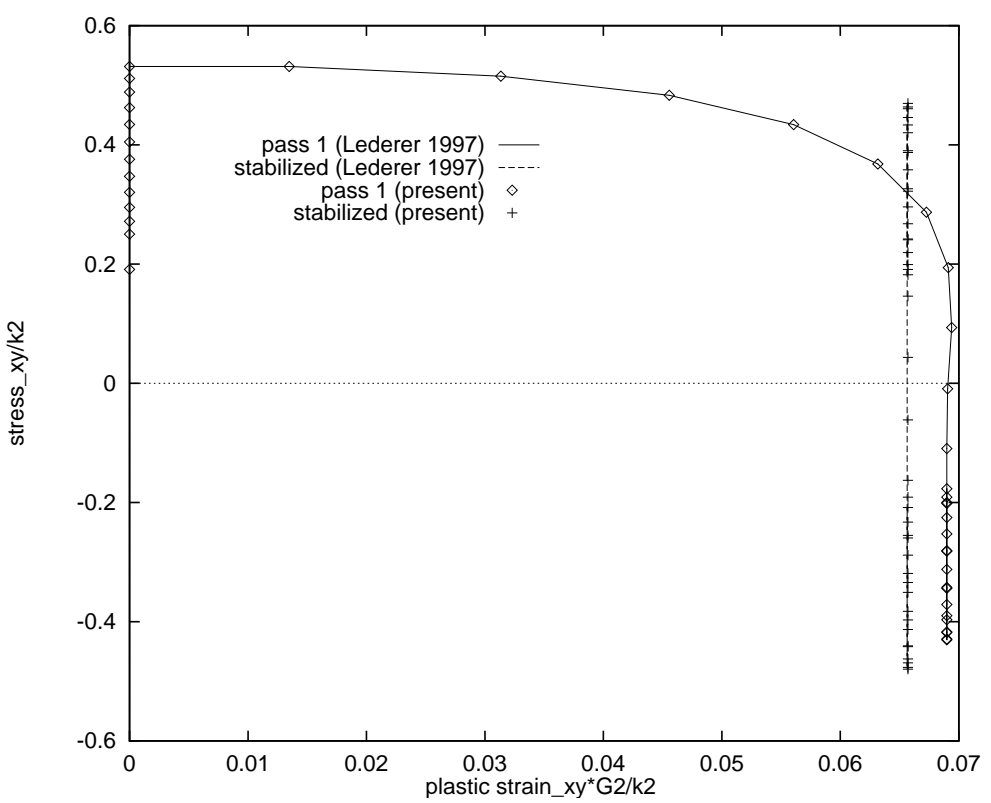

Fig. 19 Example 3: stress-strain loops produced by successive passes at depth $x=1.15 a(y=0.0)$ for $P / k=5.0$ and $\mu=0.0$ 


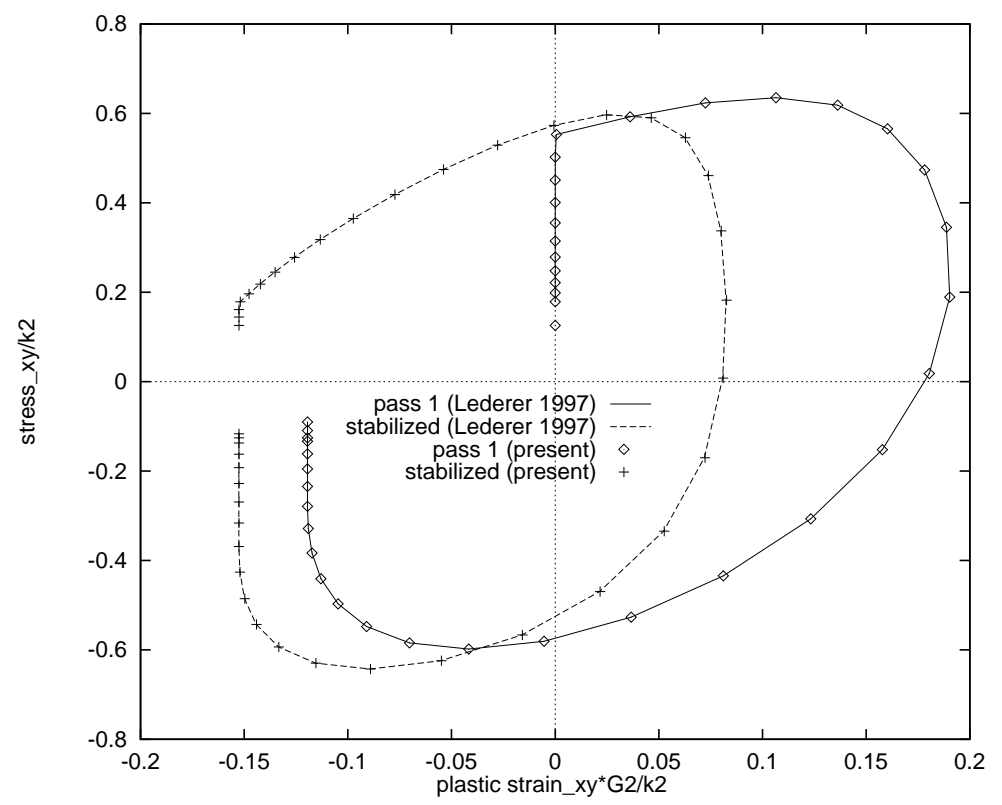

Fig. 20 Example 3: stress-strain loops produced by successive passes at depth $x=0.85 a(y=0.0)$ for $P / k=5.0$ and $\mu=0.0$

\section{Conclusion}

An integral boundary-domain formulation for steady-state elastoplastic contact over a coated half-space has been obtained. Since the homogeneous half-space fundamental solution was used, new singular domain integrals over the coating arise. Their regularization is addressed, resulting in overall weakly singular integral equations. The presented formulation has been demonstrated on numerical examples involving elastic con-

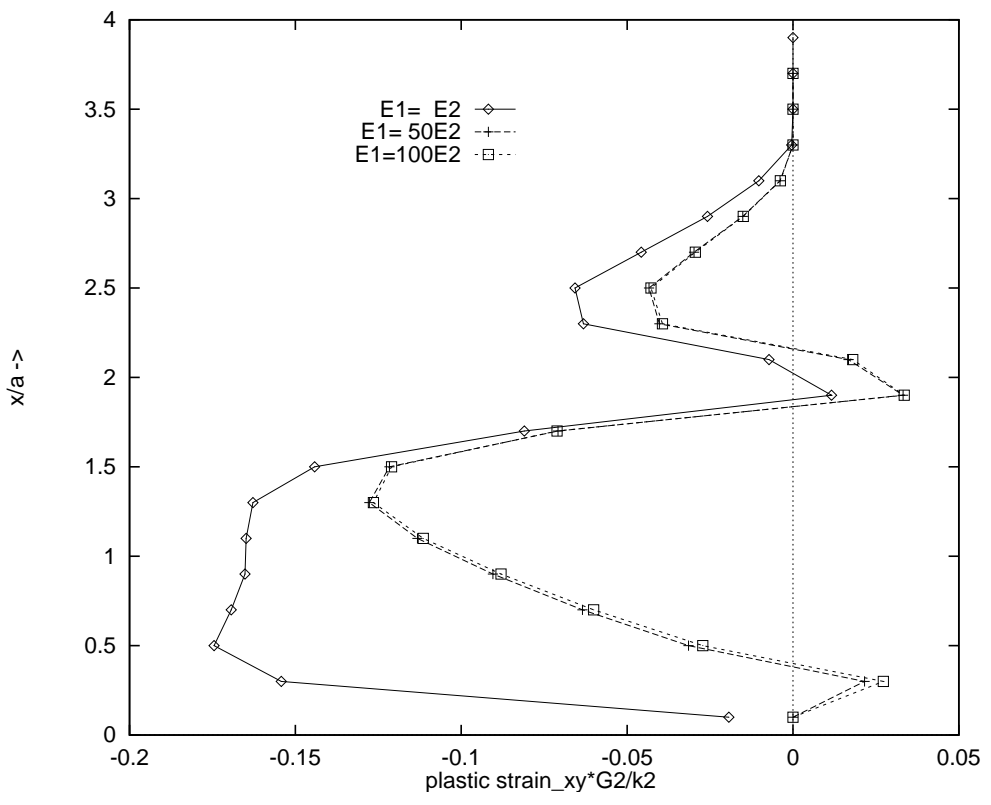

Fig. 21 Example 3: normalized plastic shear strain versus depth for $y=0.0$ 


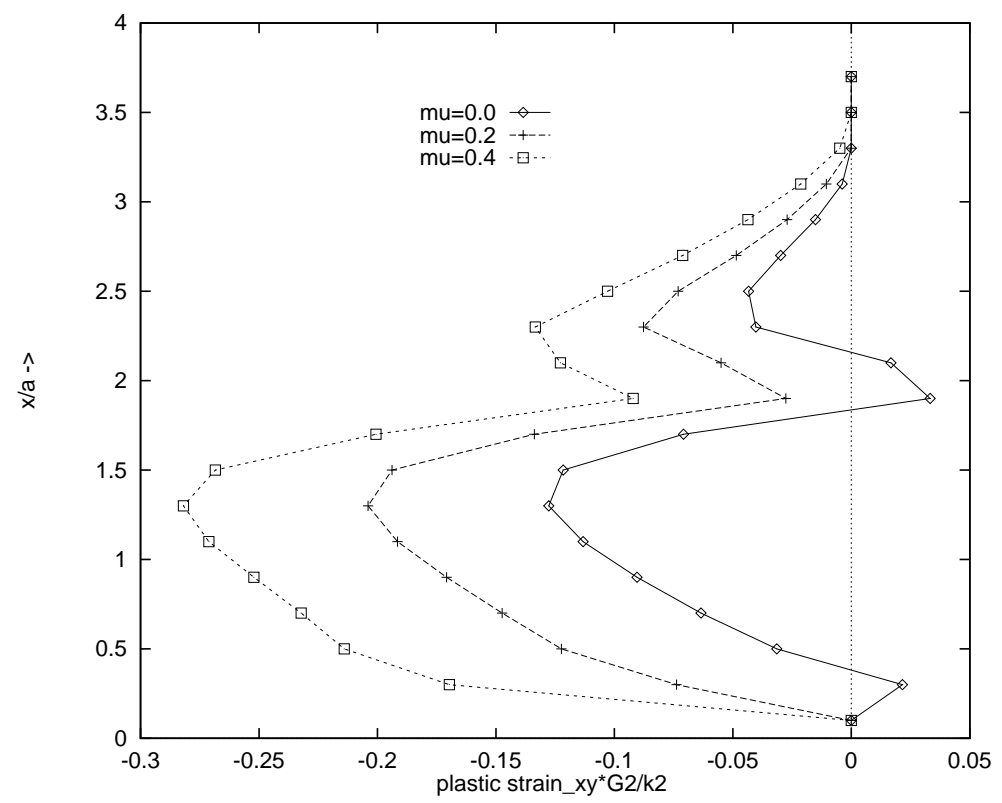

Fig. 22 Example 3: normalized plastic shear strain versus depth for $y=0.0$

tact and elastoplastic analysis for both fixed or moving Hertz loads. Numerical results compare satisfactorily with other published results when available. Some discrepancies with FEM computations on truncated domains appear to be attributable to the significant influence on stresses of the presence of nonzero strains up to infinity, which are not taken into account in the FEM simulations.

At this point, emphasis has been put on modelling a coated half-space, under either elastic or elasticplastic conditions. In particular, care has been taken in implementing a constitutive integration algorithm

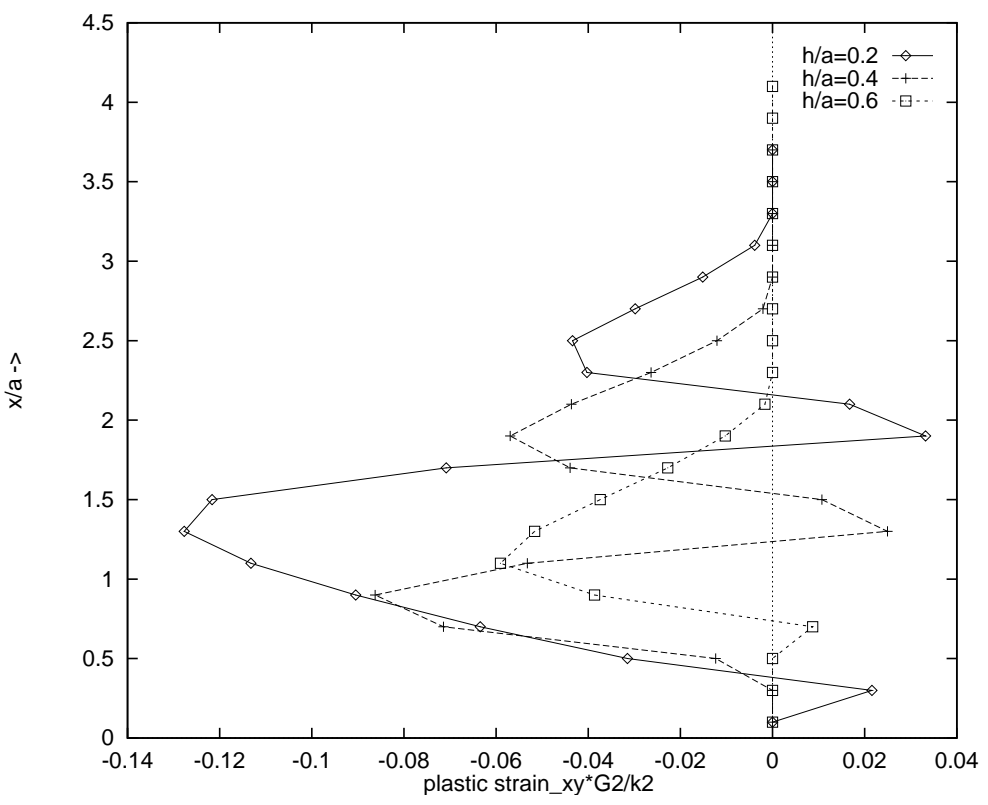

Fig. 23 Example 3: normalized plastic shear strain versus depth for $y=0.0$ 
in the case of loads moving on elastic-plastic media. On the other hand, contact conditions in the case of moving loads are, as of yet, treated under symplifying assumptions (e.g. Hertzian loads assumed). Likewise, at present, the redistribution of contact loading induced by plasticity is not taken into account. Future work aimed at improving the accuracy of our approach includes implementing a treatment of contact similar to that proposed in Kalker (1990) and Gonzalez and Abascal (1998) for elastic rolling and incorporating the coupling between contact and plasticity effects. In addition, the integral formulation presented may also be implemented for three-dimensional steady-state elastoplastic rolling/sliding analyses.

\section{References}

Antes, H., Steinfeld, B., Tröndle, G. (1991). Recent developments in dynamic stress analyses by time domain bem. Engng. Anal. with Bound. Elem., 8, 176-184.

Armstrong, P. J., Frederick, C. O. (1966). A mathematical representation of the multiaxial bauschinger effect. C.E.G.B. Report RD/B/N 731.

Balas, J., Sladek, J., Sladek, V. (1989). Stress analysis by boundary element methods. Elsevier.

Ballard, P., Constantinescu, A. (1994). On the inversion of subsurface residual stresses from surface stress measurements. J. Mech. Phys. Solids, 42, 1767-1787.

Bhargava, V., Hahn, G. T., Rubin, C. (1985a). An elastic-plastic finite element model of rolling contact. part i: analysis of single contacts. ASME J. Appl. Mech., 52, 67-74.

Bhargava, V., Hahn, G. T., Rubin, C. (1985b). An elastic-plastic finite element model of rolling contact. part ii: analysis of multiple contacts. ASME J. Appl. Mech., 52, 75-82.

Bhargava, V., Hahn, G. T., Rubin, C. (1988). Analysis of rolling contact with kinematic hardening for rail steel properties. Wear, 122, 267-283.

Bonnet, M. (1999). Boundary integral equations methods for Solids and Fluids. John Wiley and sons.

Bonnet, M., Mukherjee, S. (1996). Implicit bem formulations for usual and sensitivity problems in elastoplasticity using the consistent tangent operator concept. Int. J. Solids Struct., 33, 4461-4480.

Dang Van, K., Maitournam, M. H. (1993). Steady-state flow in classical elastoplasticity: applications to repeated rolling and sliding contact. J. Mech. Phys. Solids, 41, 1691-1710.

De Borst, R., Feenstra, P. (1990). Studies in anisotropic plasticity with reference to the hill criterion. Int. J. Num. Meth. in Eng., 29, 315-336.

Gonzalez, J. A., Abascal, R. (1998). Using the boundary element method to solve rolling contact problems. Engng. Anal. with Bound. Elem., 21, 385-392.

Huesmann, A., Kuhn, G. (1995). Automatic load incrementation technique for plane elastoplastic frictional contact problems using boundary element method. Computers and Structures, 56, 733-744. 
Johnson, K., Jefferis, J. (1963). Plastic flow and residual stresses in rolling and sliding contact. In Proc. Inst. Mech. Eng. Symp. on Rolling Contact Fatigue, pages 50-61.

Kalker, J. J. (1990). Three dimensional elastic bodies in elastic contact. Kluwer Academic Publishers.

Lederer, G., Bonnet, M., Maitournam, H. (1998). Modélisation par équations intégrales du frottement sur un demi-espace élasto-plastique. Rev. Eur. Elements finis, 7, 131-147.

Merwin, J., Johnson, K. (1963). Plastic flow and residual stresses in rolling and sliding contact. Proc. Inst. Mech. Engrs., 177, 676-685.

Mukherjee, S., Chandra, A. (1987). Nonlinear solid mechanics. In D. E. Beskos, ed., Boundary element methods in mechanics., vol. 1, pages 285-332. North Holland.

Mustoe, G. (1984). Advanced integration schemes over boundary elements and volume cells for two and three dimensional nonlinear analysis. In P. Banerjee, S. Mukherjee, eds., Developments in Boundary Element Methods - 3, pages 213-270.

Pan, E., Chen, C., Amadei, B. (1997). A bem formulation for anisotropic half-plane problems. Engng. Anal. with Bound. Elem., 20, 185-195.

Sakae, C., Keer, L. M. (1997). Application of direct method for a nonlinear kinematic hardening material under rolling/sliding line contact: constant ratchetting rate. J. Mech. Phys. Solids, 45, 1577-1594.

Simo, J. C., Taylor, R. L. (1985). Consistent tangent operators for rate-independent elastoplasticity. Comp. Meth. in Appl. Mech. Engng., 48, 101-118.

Tanaka, M., Sladek, V., Sladek, J. (1994). Regularization techniques applied to boundary element methods. Appl. Mech. Rev., 47, 457-499.

Telles, J. C. F., Brebbia, C. A. (1981). Boundry element solution for half-plane problems. Int. J. Solids Struct., 17, 1149-1158.

Yu, M., Moran, B., Keer, L. M. (1993). A direct analysis of two-dimensional elastic-plastic rolling contact. J. Tribology, 115, 227-236.

Zarka, J., Casier, J. (1979). Cyclic loading on an elastoplastic structure - practical rules. In S. Nemat-Nasser, ed., Mechanics today, 6. 


\section{List of Figures}

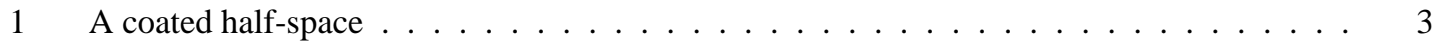

2 Plastic inclusions in the half-space . . . . . . . . . . . . . . . 9

3 Stresses produced by the inclusion of figure 2(a): comparison of analytical and numerical

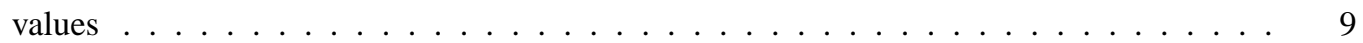

4 Stresses produced by the inclusion of figure 2(b): comparison of analytical and numerical

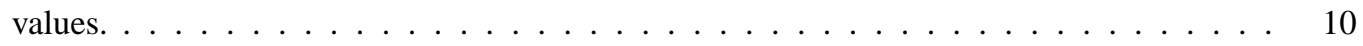

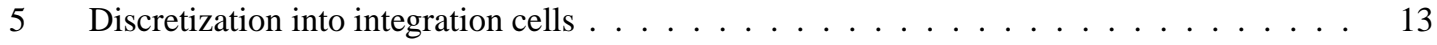

6 A roller over a coated half-space . . . . . . . . . . . . . . . . . 15

7 Indentation of a coated half-space by an elastic punch . . . . . . . . . . . . 16

8 Example 1: effect of the coating stiffness on normal contact stress _ . . . . . . . . 17

9 Example 1: effect of the coating thickness on the normal contact stress . . . . . . . . 17

10 Example 1: effect of the friction coefficient on the normal contact stress _ . . . . . 18

11 Example 1: effect of the friction coefficient on the tangential contact stress . . . . . . 18

12 Example 2: effect of the coating stiffness on plastic shear strain (plotted against $x$ for $y=$ $0.1 a)$, with $h=0.2 a \ldots \ldots \ldots \ldots \ldots$

13 Example 2: effect of the coating thickness on plastic shear strain (plotted against $x$ for

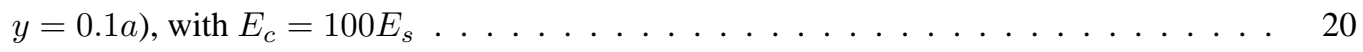

14 Example 2: effect of the frictional coefficient on plastic shear strain $(y=0.0) \ldots 21$

15 Example 2: plastic distribution along the $x$-direction $(y=0.1 a)$ for $P / k=4.5$ and $\mu=0.0 \quad 21$

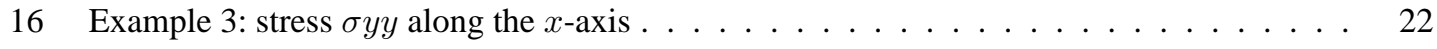

17 Example 3: constant plastic strain zones in half-plane . . . . . . . . . . . . . . . 22

18 Example 3: stress $\sigma y y$ along the $x$-axis from two unlimited constant plastic strains $\ldots \ldots 23$

19 Example 3: stress-strain loops produced by successive passes at depth $x=1.15 a(y=0.0)$

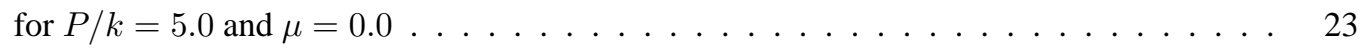

20 Example 3: stress-strain loops produced by successive passes at depth $x=0.85 a(y=0.0)$ for $P / k=5.0$ and $\mu=0.0 \ldots \ldots \ldots \ldots \ldots \ldots \ldots$

21 Example 3: normalized plastic shear strain versus depth for $y=0.0 \ldots \ldots 24$

22 Example 3: normalized plastic shear strain versus depth for $y=0.0 \ldots \ldots 25$

23 Example 3: normalized plastic shear strain versus depth for $y=0.0 \ldots \ldots 25$ 